\title{
Coding of Sound Envelopes by Inhibitory Rebound in Neurons of the Superior Olivary Complex in the Unanesthetized Rabbit
}

\author{
Shigeyuki Kuwada and Ranjan Batra \\ University of Connecticut Health Center, Department of Anatomy, Farmington, Connecticut 06030-3405
}

\begin{abstract}
Most natural sounds (e.g., speech) are complex and have amplitude envelopes that fluctuate rapidly. A number of studies have examined the neural coding of envelopes, but little attention has been paid to the superior olivary complex (SOC), a constellation of nuclei that receive information from the cochlear nucleus. We studied two classes of predominantly monaural neurons: those that displayed a sustained response to tone bursts and those that gave only a response to the tone offset. Our results demonstrate that the off neurons in the SOC can encode the pattern of amplitude-modulated sounds with high synchrony that is superior to sustained neurons. The upper cutoff frequency and highest modulation frequency at which significant synchrony was present were, on average, slightly higher for off neurons compared with sustained neurons. Fi-
\end{abstract}

Most natural sounds (e.g., speech) have envelopes that over time fluctuate in both frequency and amplitude. Consequently, considerable attention has been devoted to the neural processing of envelopes. Studies of envelope coding of monaural sounds have concentrated on the auditory nerve (Palmer, 1982; Joris and Yin, 1992), cochlear nucleus (CN) (Moller, 1974, 1976; Frisina et al., 1990; Kim et al., 1990; Rhode, 1994; Rhode and Greenberg, 1994a), inferior colliculus (Rees and Moller, 1987; Langner and Schreiner, 1988; Batra et al., 1989; Rees and Palmer, 1989; Heil et al., 1995), auditory thalamus (Creutzfeldt et al., 1980; Rouiller et al., 1981), and auditory cortex (Creutzfeldt et al., 1980; Schreiner and Urbas, 1986, 1988; Eggermont, 1993). However, little attention has been paid to envelope coding in monaural neurons of the superior olivary complex (SOC), a constellation of nuclei that receives information from the ventral $\mathrm{CN}$ and projects this information to higher structures (Schwartz, 1992; Warr, 1992; Helfert and Aschoff, 1997) as well as provides feedback to the CN and the cochlea (Liberman and Brown, 1986). The few studies of envelope coding in the SOC either focused on structures involved in binaural processing [lateral superior olive (LSO) and medial superior olive (MSO)] (Grothe et al., 1997; Batra et al., 1997b; Joris and Yin, 1998) or were conducted in the mustached bat, which has a system specialized for echolocation (Grothe, 1994). However, the SOC does contain many neurons dedicated to monaural processing. Most of these neurons are located in periolivary regions that lie outside the primary binaural nuclei, i.e., the LSO and MSO (Guinan et al., 1972a,b; Tsuchitani, 1977). In this

\footnotetext{
Received Sept. 16, 1998; revised Dec. 29, 1998; accepted Dec. 31, 1998.

This work was supported by National Institutes of Health Grants DC02178 and NS18027. We thank Lisa Seman for technical assistance and Douglas C. Fitzpatrick and Bill D'Angelo for helpful comments.

Correspondence should be addressed to Dr. Shigeyuki Kuwada, Department of Anatomy, University of Connecticut Health Center, Farmington, CT 06030-3405. Copyright (C) 1999 Society for Neuroscience $\quad 0270-6474 / 99 / 192273-15 \$ 05.00 / 0$
}

nally, most sustained and off neurons encoded the level of pure tones over a wider range of intensities than those reported for auditory nerve fibers and cochlear nucleus neurons. A traditional view of inhibition is that it attenuates or terminates neural activity. Although this holds true for off neurons, the robust discharge when inhibition is released adds a new dimension. For simple sounds (i.e., pure tones), the off response can code a wide range of sound levels. For complex sounds, the off response becomes entrained to each modulation, resulting in a precise temporal coding of the envelope.

Key words: auditory system; amplitude modulation; single unit recording; monaural signal processing; envelope coding; intensity coding

study, we investigated the ability of neurons in the SOC of the unanesthetized rabbit to code the envelopes of monaural signals.

\section{MATERIALS AND METHODS}

Experimental preparation. Almost all previous studies of envelope coding have used an anesthetized preparation. We used unanesthetized DutchBelted rabbits $(n=9)$ because anesthetics are known to alter auditory responses (Evans and Nelson, 1973; Young and Brownell, 1976; Brownell et al., 1979; Ritz and Brownell, 1982; Kuwada et al., 1989). We chose the rabbit because it adapts well to the restraint required when an unanesthetized preparation is used. With light restraint and little previous training, most rabbits will remain still for a period of 2 or more hours. Additionally, the rabbit has a well-developed SOC (Ramón y Cajal, 1909) and auditory structures with sizes that lie in the middle of the range of all mammals studied (Glendenning and Masterton, 1995).

Surgery and recording procedures. Our surgical procedures have been described previously (Batra et al., 1997a) and conformed to the National Institutes of Health guidelines and protocols approved by the Animal Care Committee of the University of Connecticut Health Center. Aseptic surgery was performed in three stages. For each stage, rabbits were anesthetized with a mixture of ketamine $(35 \mathrm{mg} / \mathrm{kg}$ ) and xylazine (5 $\mathrm{mg} / \mathrm{kg}$ ) delivered intramuscularly. In the first stage, skin and muscle were retracted to expose a dorsal region of the skull. Dental acrylic and screws threaded into the skull were used to affix a brass rod, parallel to the sagittal suture, and slightly to its left. The right side of the skull was left exposed between bregma and lambda. The rabbit was then allowed several days to recover. In the second stage, the rabbit was fitted with custom ear molds for sound delivery. An ear mold was made by first inserting a metal tube into the ear canal and then pressing ear impression compound (Audalin; Esschem, Essington, PA) around it. Once the mold had been removed from the ear, the metal tube was extracted and replaced with a sound delivery tube made from electrical shrink tubing. The tip of the sound delivery tube reached to within $2 \mathrm{~cm}$ of the tympanum. After a 1-2 d recovery period, the rabbit was placed in a soundproof booth and acclimated to head and body restraint and to the ear molds. The rabbit's body was restrained by a snug-fitting body stocking and placed in a Plexiglass couch with nylon safety straps. The head was held stationary by clamping the brass rod. Ear molds were inserted and tightly coupled to earphones (Beyer DT-48). Daily sessions were gradually increased to $2 \mathrm{hr}$. The acclimation period lasted 1-2 
weeks. After this period, the third stage of surgery was performed. A small hole $(2-4 \mathrm{~mm})$ was made in the skull, just rostral to lambda. The exposed dura was rinsed with sterile saline, treated with a topical antibiotic (Bacitracin), and covered with sterilized elastopolymer.

During the recording session the animal was restrained as described above. The elastopolymer cap was removed, and the exposed dura was desensitized with a topical anesthetic (Lidocaine). The dura was pierced with a thin-wall hypodermic needle (23xx gauge) inside which rode the microelectrode (glass-coated platinum-iridium or platinum-tungsten). A Burleigh microdrive advanced the microelectrode. Electrode advancement, acoustic stimulation, and data collection were controlled from outside the soundproof booth. Neural recordings were filtered between 300 and $3000 \mathrm{~Hz}$ and amplified 5,000-10,000 times. Action potentials were timed with an accuracy of $10 \mu \mathrm{sec}$.

Typically, a rabbit participated in daily recording sessions over a period of $\sim 2-3$ months. Each session lasted $\sim 2 \mathrm{hr}$. Although we did not measure the rabbit's exact behavioral state (e.g., asleep, awake, drowsy), it was monitored with a video camera, and the session was terminated if the rabbit fidgeted. The rabbit's comfort was a priority for ethical as well as for practical reasons, because any movement would disrupt neural recordings.

Acoustic stimulation and calibration. Stimuli were generated by dual digital stimulators (Rhode, 1976). All stimuli were gated with linear rise-fall times of $4.0 \mathrm{msec}$. Intensity levels were initially referenced to a standard calibration and later corrected using the actual calibration for that animal. In earlier experiments, acoustic calibration was performed after the animal was killed. In later experiments, the calibrations were performed under deep anesthesia. The calibration procedure is described in detail in Batra et al. (1997a). Acoustic cross talk between the ears was measured by delivering tones to one ear while recording the acoustic signal in the other ear. The level in the unstimulated ear was $\sim 55-60 \mathrm{~dB}$ below that in the stimulated ear from $0.1-3 \mathrm{kHz}$. Above 3 $\mathrm{kHz}$, the cross talk was smaller than the noise level (i.e., not measurable).

Testing procedure. A neuron's best frequency was assessed by collecting the responses to short $(50 \mathrm{msec})$, monaural tone bursts between 0.2 and $35 \mathrm{kHz}$ in 0.5 octave steps at a suprathreshold level [typically $50-70 \mathrm{~dB}$ sound pressure level (SPL)]. For sustained neurons, the best frequency was defined as the frequency at which the sustained response was maximal, whereas for off neurons it was the frequency at which the off response was maximal. The rate versus level function and discharge pattern were then determined using short, best-frequency tone bursts (100-125 msec repetition interval, 50-100 repetitions, typically 20-70 dB SPL).

We usually determined whether a neuron was binaural by delivering best-frequency tone bursts to the preferred ear while varying the level to the other ear. Another way was to separately test each ear over a range of frequencies. If the binaural response resembled the monaural response to the preferred ear, or if it could be attributed to the effects of acoustic cross talk, then the neuron was classified as monaural. Conversely, if the binaural response differed systematically from the monaural response to the preferred ear, or if its response to the other ear could not be attributed to cross talk (e.g., responses to the preferred ear were excitatory and the responses to the other ear were inhibitory), then the neuron was classified as binaural. Neurons were also tested for binaural inputs by examining their sensitivity to interaural time difference using the binaural beat stimulus, i.e., a small frequency difference between the carrier or envelope frequencies between the two ears (Kuwada et al., 1979; Batra et al., 1989). The responses of SOC neurons sensitive to interaural time differences were published previously (Batra et al., 1997a,b) and are not included in this study.

Sinusoidally amplitude-modulated (SAM) tones were 1-1.1 sec in duration, with a repetition interval of 1.2 or $1.3 \mathrm{sec}$. At each modulation frequency, two to three repetitions of the SAM tone were presented. The carrier was held at the neuron's best frequency, and modulation depth was initially set at $80 \%$. Modulation frequencies between 25 and $800 \mathrm{~Hz}$ were routinely tested, and higher frequencies were tested if necessary. Conditions permitting, we tested the response to SAM tones over a range of levels and modulation depths.

Data analysis. The regularity of the discharge pattern to short tone bursts for sustained neurons was assessed by using the coefficient of variation $(\mathrm{CV})$. We determined the average $\mathrm{CV}$ by dividing the mean of the interspike intervals (ISIs) over the tone bursts by the SD.

The dynamic range of a neuron to tones, i.e., the range of intensities over which the response increased monotonically, was estimated from its rate-level function. The analysis window was the stimulus duration for sustained neurons and was the off interval for off neurons. The lower limit of the dynamic range (i.e., threshold) was neural threshold, i.e., the lowest intensity that evoked a significant response. Threshold was determined using the "repetition interval synchronization coefficient" (Batra et al., 1997a). This coefficient was calculated by treating the repetition interval of the tone as the period of a cyclic stimulus. A synchronization coefficient that was significant (Rayleigh test of uniformity; $p<0.025$ ) (Mardia, 1972) denoted a response that followed the stimulus. The upper limit of the dynamic range was the intensity at which the response ceased to increase systematically with intensity, i.e., saturation. This limit was assessed visually. The dynamic range was the difference between the two limits. For many neurons, the range of intensities that was used did not encompass the lower or upper limit or both, so the dynamic range for many neurons was likely underestimated.

Phase-locking (i.e., synchrony) to the modulation frequency was assessed both visually and quantitatively. Visual assessment was performed using period histograms, which depict the average response over a cycle of the modulation period. The first $100 \mathrm{msec}$ of the response was excluded from the analysis to avoid onset effects. Strength of synchrony $(R)$ was determined by calculating the vector strength of the response (Goldberg and Brown, 1969; Kuwada et al., 1987). A value of $R=1$ indicates perfect synchrony, and a value of $R=0$ indicates no synchrony. The phase of the modulation at which the neuron discharged was estimated using the mean phase of the response. Only responses that were significantly synchronized are displayed (Rayleigh test of uniformity; $p<$ 0.001) (Mardia, 1972), except where noted.

Localization of recording sites. For each electrode penetration, the electrode was positioned relative to a reference mark on the skull. The depth at which each neuron was studied was noted. During the last recording session, electrolytic lesions were made at selected sites $(10 \mu \mathrm{A}$ for $10-20 \mathrm{sec})$. Four to six days after lesion, the animals was deeply anesthetized with sodium pentobarbital, and most were perfused with a $10 \%$ solution of formol saline. In some animals, the brain was fixed by immersion in a $10 \%$ formol-saline solution. The brains were frozen and sectioned $(30$ or $60 \mu \mathrm{m})$ in the plane of the electrode penetrations and then stained with cresyl violet or thionin (Kuwada et al., 1987).

\section{RESULTS}

Our results are based on the responses of 65 neurons in the SOC. We classified these neurons into sustained $(n=42)$ or off $(n=23)$ on the basis of their discharge pattern (see Materials and Methods). Onset responses constituted a small part of our sample and were not considered further. The best frequencies of sustained and off neurons were similar and ranged from 0.75 to $35 \mathrm{kHz}$, with a mode between 8 and $9 \mathrm{kHz}$.

Of the 35 sustained neurons tested for binaural influences, most were monaural $(29 / 35 ; 83 \%)$. Of the monaural neurons, approximately half $(15 / 29)$ responded to contralateral stimulation, and the remainder responded to ipsilateral stimulation. The remaining six sustained neurons exhibited binaural influences (see Materials and Methods). Two of these respond more strongly to contralateral stimulation. To ipsilateral stimulation, one showed a sustained suppression of spontaneous activity, and the other showed a transient excitation. The other four preferred ipsilateral stimulation. To contralateral stimulation, two showed a sustained excitation, and two displayed a transient suppression. Most off neurons tested for binaural influences were monaural $(13 / 18 ; 72 \%)$, and all responded to contralateral stimulation. The five binaural off neurons $(28 \%)$ all preferred contralateral stimulation. To ipsilateral stimulation, two responded with sustained excitation, another responded with a transient excitation, another with a transient excitation followed by a sustained suppression, and yet another with a sustained suppression of spontaneous activity.

\section{Location of recording sites}

Our procedure for estimating the location of a neuron was subject to considerable error because recordings made over the course of months were referenced to electrolytic marking lesions made at 
the end of this period. Thus brain movements and any slight day-to-day variations in electrode positioning could influence the estimated location of a neuron. Another factor was the large distance $(\sim 14-18 \mathrm{~mm})$ the electrode traversed to reach the SOC. Slight deviations in straightness of the electrode or the guide tube in which it rode could lead to large errors in the intended location (Batra et al., 1997a). With these limitations in mind, we found that all but two of the off neurons were located medial to the LSO. The off neurons were scattered in the dorsal-ventral plane that extended above and below the MSO. Sustained neurons that responded preferentially to stimulation of the contralateral ear were primarily located medial and ventral to the LSO. In contrast, the sustained neurons that preferred ipsilateral stimulation were located almost exclusively near the ventral border of the LSO.

The sustained and off neurons were likely not located in the principal binaural nuclei, i.e., LSO and MSO. Previously, we reported on neurons in the SOC that were sensitive to interaural time differences. Most of these were presumed to be located in the LSO and MSO (Batra et al., 1997a). These neurons were excluded from the present sample. The neurons sensitive to interaural time differences were recorded in 86 electrode penetrations. The sustained and off neurons in the present sample were recorded in 53 electrode penetrations. There were only five electrode penetrations that were common to the present sample and those in which neurons sensitive to interaural time differences were encountered. Moreover, in these common penetrations, sustained and off neurons were encountered ventral to the neurons sensitive to interaural time differences.

\section{Responses to tones}

Sustained neurons displayed various discharge patterns in response to short tone bursts (Fig. $1 A-D$ ) that were similar to those reported previously in the CN (for review, see Rhode and Greenberg, 1992). Some neurons displayed a tightly locked onset response followed by peaks throughout the stimulus that were separated by regular intervals, i.e., a chopping pattern (Fig. 1A). In other neurons, the onset was followed by a pause and then a chopping response that persisted for only a short time (Fig. 1B). Some neurons displayed a pauser pattern, i.e., a tightly locked onset, followed by a pause that was then followed by a sustained discharge for the duration of the tone burst (Fig. 1C). Finally, there were neurons with primary-like discharge patterns consisting of a prominent onset that smoothly decayed to a steady-state level (Fig. 1D).

A common feature of many sustained neurons was the regularity of their discharge. Regularity was assessed by examining the $\mathrm{CV}$ (see Materials and Methods) at the intensity that elicited the strongest discharge. The neurons of Figure $1, A$ and $B$, had CVs $<0.5$, which was consistent with their sustained or transient chopping pattern. The pauser neuron (Fig. $1 C$ ) had an intermediate CV (0.50), whereas the primary-like neuron (Fig. 1C) had an irregular firing pattern $(\mathrm{CV}=0.81)$. Most sustained neurons $(31 / 42 ; 74 \%)$ had a regular firing pattern.

The off discharge pattern has rarely been reported in the $\mathrm{CN}$ but was encountered routinely in the SOC. Off neurons displayed a complete or almost complete absence of activity during the tone burst but discharged at the tone offset (Fig. $1 E-H$ ). Spontaneous activity was almost always suppressed during the tone (Fig. $1 E, G, H)$. The magnitude of the off response differed among neurons. Some neurons had relatively weak responses (Fig. 1E), whereas others had relatively strong responses (Fig. $1 H$ ). Off neurons also differed in the pattern of their responses. Some responded with a single peak that decayed to spontaneous levels by $\sim 50 \mathrm{msec}$ after tone offset (Fig. $1 F, G$ ), whereas others responded with two peaks separated by a pause (Fig. $1 E, H$ ). For both types of off responses, each peak in the poststimulus time histogram (PST) consisted of multiple action potentials.

Sustained neurons had dynamic ranges that were larger than those of auditory nerve fibers and neurons in the CN. For auditory nerve fibers and neurons in the $\mathrm{CN}$, the dynamic range is typically 10-40 dB SPL (Rhode and Greenberg, 1994b). Rateintensity functions illustrating the dynamic range of neurons in the SOC are shown in Figure 2 (same neurons as Fig. 1). The responses of the majority of sustained neurons $(27 / 31 ; 87 \%)$ increased monotonically with stimulus level, but the dynamic range of these neurons varied considerably (Fig. $2 A$ ). Some had relatively small dynamic ranges (Fig. $2 A$; neuron $D, \sim 30 \mathrm{~dB}$ ), whereas others had much larger dynamic ranges (Fig. $2 A$; neurons $A$ and $B,>50 \mathrm{~dB}$ ). The average dynamic range of sustained neurons was $37 \pm 11 \mathrm{~dB}$ (mean $\pm \mathrm{SD} ; n=31)$, with most of the neurons $(55 \%)$ having a dynamic range $\geq 40 \mathrm{~dB}$. Approximately $19 \%$ had dynamic ranges $>50 \mathrm{~dB}$. For many neurons, their dynamic range was probably underestimated (see Materials and Methods).

Off neurons also had dynamic ranges that were larger than auditory nerve fibers or $\mathrm{CN}$ neurons (Fig. $2 B$ ). As with sustained neurons, some off neurons had relatively small dynamic ranges (data not illustrated), whereas others had much larger dynamic ranges (Fig. $2 B$; all $>50 \mathrm{~dB}$ ). The average dynamic range for off neurons was $42 \pm 12 \mathrm{~dB}$. Almost all off neurons (16/17; 94\%) showed a monotonic increase in discharge rate with stimulus level and most $(12 / 17 ; 71 \%)$ had a dynamic range $\geq 40 \mathrm{~dB}$ SPL. About half $(8 / 17 ; 47 \%)$ had dynamic ranges $>50 \mathrm{~dB}$.

\section{Responses to SAM tones: modulation transfer functions}

To determine the ability of sustained and off neurons to encode the envelopes of complex sounds, we measured their discharge rate and response synchrony to SAM tones over a range of modulation frequencies. Although the activity of off neurons was suppressed during a tone burst, they discharged continuously to SAM tones at lower modulation frequencies. Figure 3 depicts PSTs of the responses of a sustained and an off neuron to SAM tones. The sustained neuron gave a sustained response at all modulation frequencies (Fig. $3 A-E$ ), similar to its response to tone bursts. The off neuron produced an off discharge at all modulation frequencies (Fig. $3 F-J$ ), but at lower frequencies there was also a sustained discharge during the SAM tone (Fig. $3 G-J$ ).

Off neurons were strongly synchronized to the envelope at all modulation frequencies at which they responded to SAM tones, whereas the synchrony of sustained neurons varied with modulation frequency. Figure 4 shows the responses of the same neurons as in Figure 3 plotted as a function of modulation phase. The sustained neuron synchronized well at intermediate modulation frequencies (Fig. 4C-E), but at higher and lower modulation frequencies the strength of synchrony declined (Fig. 4A,B,F). In contrast, the off neuron synchronized strongly at all (Fig. $4 H-L$ ) but the highest modulation frequency (Fig. 4G), although the discharge declined at higher frequencies (Fig. 4G,H).

The contrasting responses of sustained and off neurons to SAM tones resulted in their having different modulation transfer functions (MTFs) (Fig. 5). For the sustained neuron of Figures 3 and 4 , the discharge rate as a function of modulation frequency (rate MTF) was relatively flat (Fig. $5 A, \square$ ), whereas for the off neuron 
Sustained Neurons
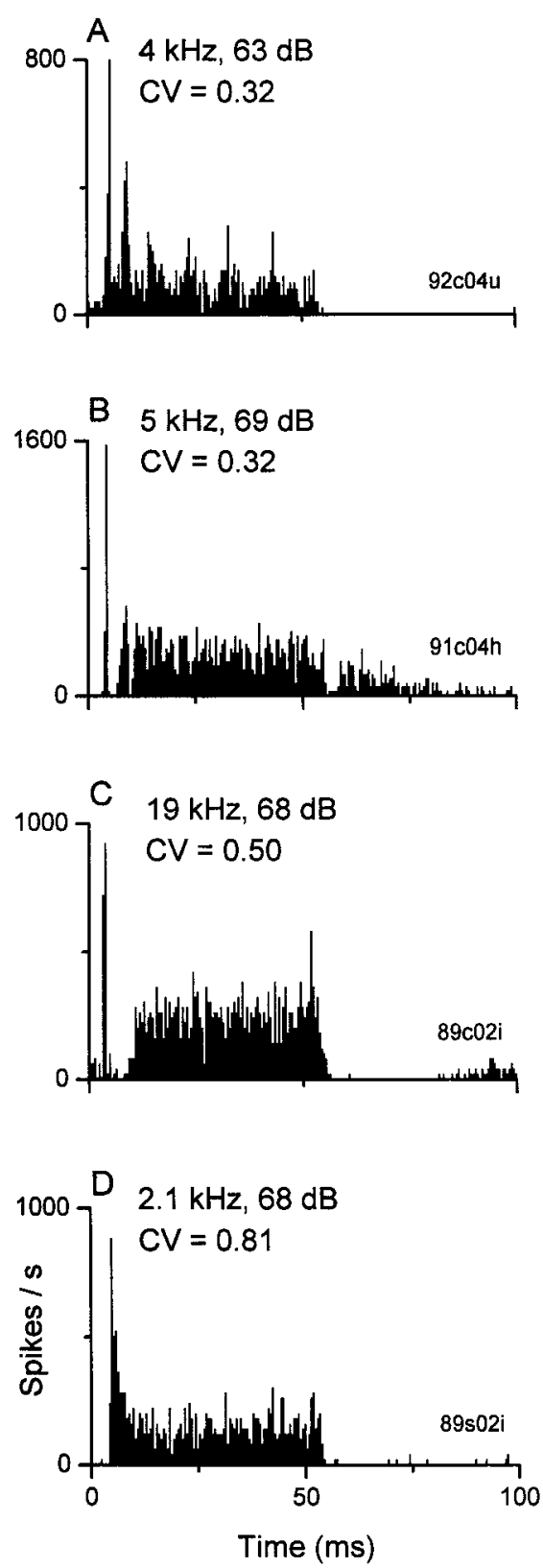

Off Neurons
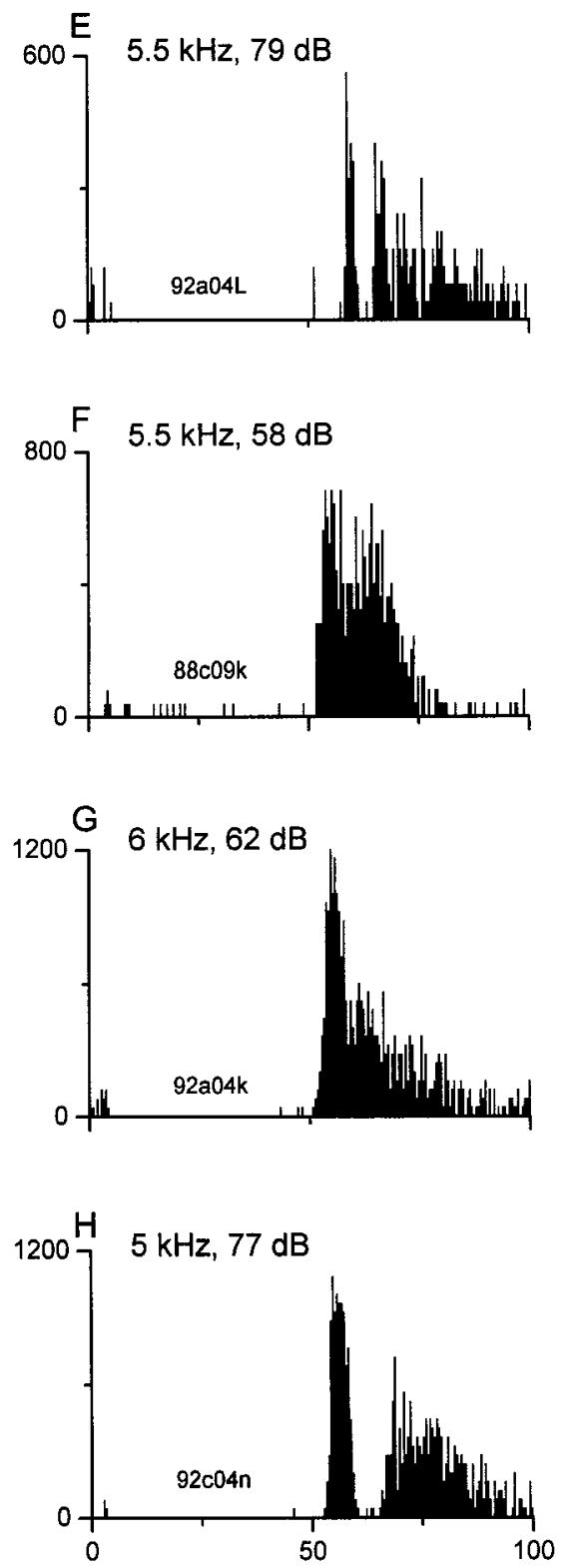

Figure 1. Examples of discharge patterns of sustained $(A-D)$ and off neurons $(E-H)$. In all cases, responses are to $50 \mathrm{msec}$ tone bursts at the frequency and intensity indicated. $A$, Repetition interval and number of repetitions, $125 \mathrm{msec} \times 100 ; B, 125$ $\mathrm{msec} \times 75 ; C, 100 \mathrm{msec} \times 100 ; D, 100 \mathrm{msec} \times 100$; $E, 125 \mathrm{msec} \times 50 ; F, 200 \mathrm{msec} \times 50 ; G, 125 \mathrm{msec} \times$ $50 ; H, 125 \mathrm{msec} \times 50$. The $\mathrm{CV}$ is indicated for sustained neurons (see Materials and Methods). Bin width is $0.5 \mathrm{msec}$.

it was low-pass (Fig. 5A, $\bigcirc$ ). The synchrony as a function of modulation frequency (synchrony MTF) was bandpass for the sustained neuron (Fig. 5B, $\mathbf{\square}$ ) but was low-pass for the off neuron (Fig. 5B, $\bigcirc$ ). At each modulation frequency, the off neuron synchronized to the envelope more strongly than the sustained neuron.

In general, the MTF patterns of the neurons illustrated in Figures 3-5 reflected the responses of our entire sample of sustained and off neurons. Figure $6 A$ shows the synchrony and rate MTFs for about half of our sustained neurons $(n=20)$. The remainder $(n=22)$ were not plotted for the sake of visual clarity. In general, the synchrony MTFs of sustained neurons were bandpass (Fig. 6A). Averaging across all neurons yielded a population MTF that was mildly bandpass (Fig. $6 B$ ). The bandpass nature of most MTFs was more clearly visible when the MTFs in Figure $6 \mathrm{~A}$ were normalized to each neuron's best modulation frequency (BMF) and its maximum synchrony (Fig. 6C). Averaging across the normalized MTFs of all the neurons yielded a population MTF based on synchrony that was clearly bandpass (Fig. 6D). In contrast the rate MTFs were relatively flat (Fig. $6 E$ ), and the corresponding population MTF was relatively flat as well (Fig. $6 F$ ). Most off neurons had synchrony and rate MTFs that were low-pass (Fig. 7, $A$ and $C$, respectively). The synchrony was usually very high $(>0.8)$ within the pass-band. The low-pass shapes of the MTFs resulted in population MTFs that were also low-pass (Fig. 7B,D).

Off neurons encoded a slightly higher range of modulation frequencies than sustained neurons. Figure 8 compares the upper cutoff modulation frequency and the highest modulation frequency at which significant synchrony was present for these two 


\section{A Sustained Neurons}
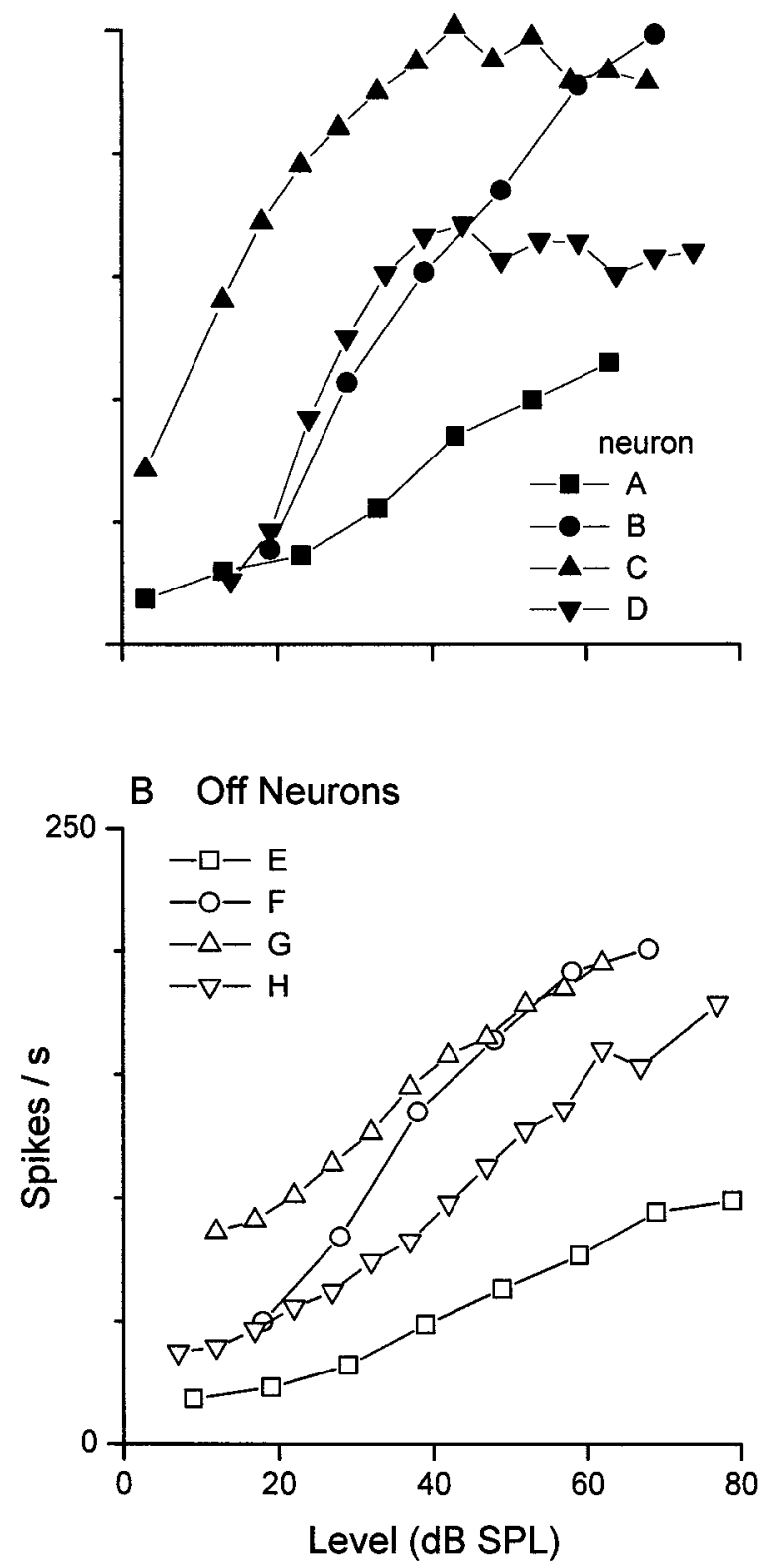

Figure 2. Sustained and off neurons can display a wide dynamic range. $A$, Discharge rate versus stimulus level for the four sustained neurons in Figure 1. $B$, Same for the four off neurons in Figure 1. Dynamic ranges for neurons $A-H$ were $60,50,40,30,50,50,50$, and $65 \mathrm{~dB}$.

groups. Figure $8 A$ displays the BMFs of sustained neurons. Because most off neurons had MTFs that were low-pass, it was inappropriate to measure their BMFs. For this reason, we estimated (by interpolation) the high modulation frequency cutoff at which the synchrony was $20 \%$ lower than the maximum (Fig. $8 B$ ). This level was chosen because at high modulation frequencies synchrony of off neurons declined abruptly to where synchrony was no longer significant. The steep decline made interpolating the cutoff frequency at a conventional level (e.g., $6 \mathrm{~dB}$ below maximum) impossible in most neurons. The same measure was made on sustained neurons. Based on this measure, off neurons synchronized, on average, to modulation frequencies $\sim 140 \mathrm{~Hz}$ higher than sustained neurons $(t=2.39$; df $=53$; $p<0.02)$. We also measured the highest modulation frequency at which significant synchrony was present (Fig. $8 C$ ). The highest modulation frequency was $\sim 80 \mathrm{~Hz}$ higher for off neurons, but this difference was not significant $(p>0.05)$.

Off neurons also displayed higher synchrony than sustained neurons. We compared the highest synchrony exhibited by neurons of each type (Fig. $8 D$ ). The highest synchrony of sustained neurons covered a wide range $(0.21-0.95)$, with an average of 0.70 . In contrast, the highest synchrony of off neurons clustered tightly between 0.83 and 0.99 with an average of 0.94 . This difference in synchrony between sustained and off neurons was highly significant $(t=6.78$; df $=71 ; p<0.001)$.

In a linear system, the phase of the synchronized response changes linearly with modulation frequency. The slope of the linear fit to this plot is an estimate of the delay between the acoustic stimulus and the responses of the neuron (Anderson et al., 1971; Joris and Yin, 1992). The intercept of the fit is an estimate of which phase of the signal reaching the neuron evokes a response. Figure $9 A$ illustrates the linear change in response phase with modulation frequency for a sustained and an off neuron. The slopes of the fits indicate delays of 5.0 and $4.7 \mathrm{msec}$ for the sustained and off neurons, respectively. The phase intercept of the sustained neuron is 0.11 cycles, and that of the off neuron is 0.71 cycles. The modulation was presented in sine phase, meaning that the peak of the envelope occurred at a phase of 0.25 cycles, and the trough at 0.75 cycles. Thus, the sustained neuron discharged just before the peak, whereas the off neuron discharged just before the trough of the incoming signal.

On average, sustained and off neurons responded with similar delays (sustained, $5.1 \pm 1.2 \mathrm{msec}$; off, $4.9 \pm 0.3 \mathrm{msec}$ ) (Fig. 9B). However, the delays of sustained neurons were distributed over a wider range than those of off neurons.

In the auditory nerve and $\mathrm{CN}$, delay decreases with characteristic frequency of the neuron. This relationship reflects the travel time along the basilar membrane. In our SOC neurons there was little if any relationship between a neuron's delay and its best frequency.

The phase intercepts of the phase-frequency plots were consistent with the notion that the response of sustained neurons is via excitation and the response of off neurons is via a rebound from inhibition (Fig. 9C). The phase intercepts of all but three of the sustained neurons were grouped slightly below 0.25 cycles, and those of off neurons were all grouped slightly below 0.75 cycles. The difference between the means was 0.57 cycles. Thus, sustained neurons responded just before the maximum amplitude of the incoming signal was reached $(0.25$ cycles $)$, and off neurons responded just before the minimum amplitude was reached $(0.75$ cycles).

\section{Effects of modulation depth}

Both sustained and off neurons synchronized to a wide range of modulation depths but differed in how the response changed with modulation depth. Figure 10 plots the discharge rate and synchrony as a function of modulation depth for four sustained and four off neurons. For comparison, we also include the minimum and maximum of rate and synchrony functions (Fig. 10, dashed lines) in a small number of auditory nerve fibers in the cat (Joris and Yin, 1992, their Fig. 2). Sustained neurons responded with 
Sustained Neuron

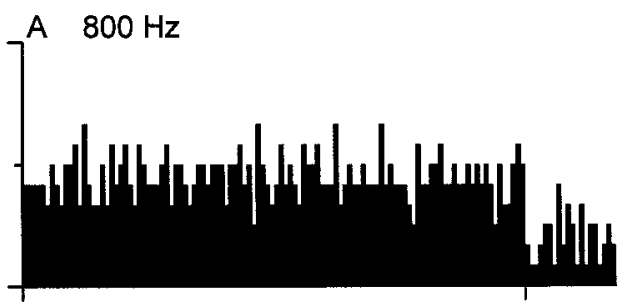

B $400 \mathrm{~Hz}$
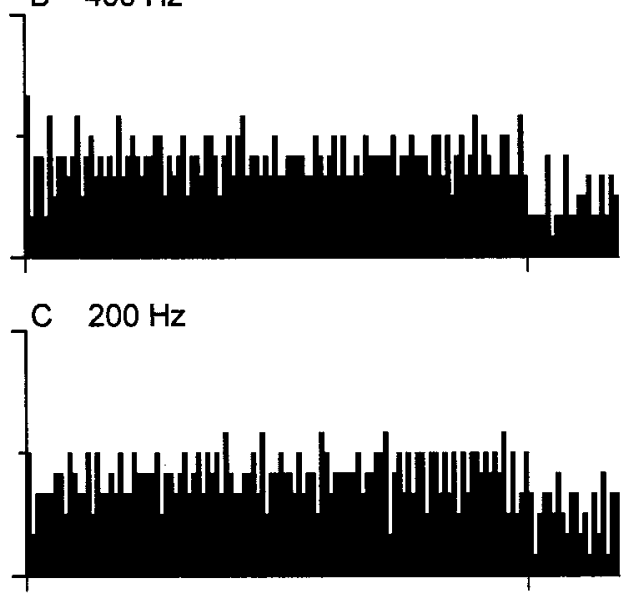

D $50 \mathrm{~Hz}$
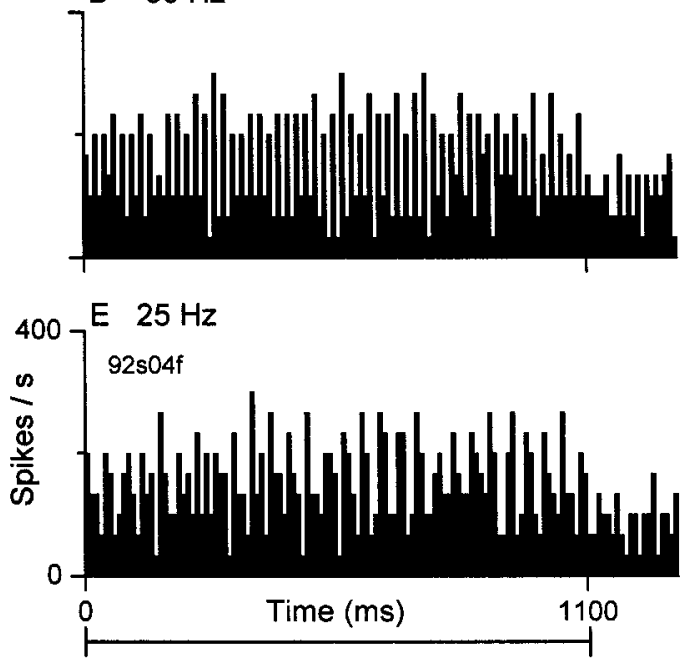

Off Neuron
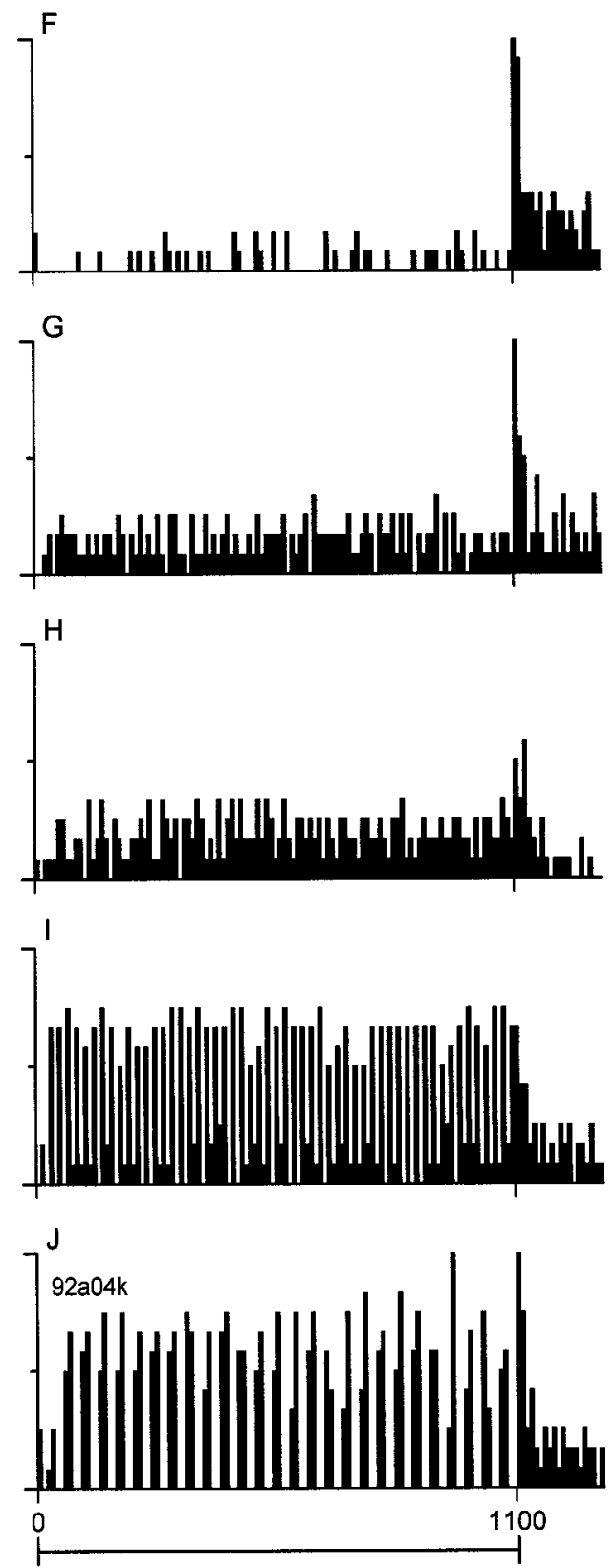

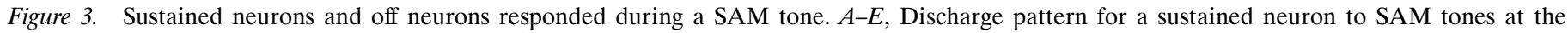

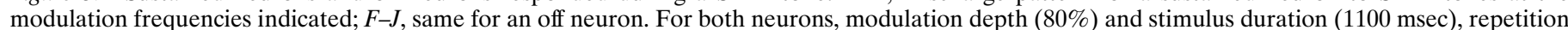

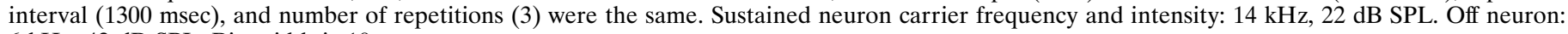
$6 \mathrm{kHz}, 42 \mathrm{~dB}$ SPL. Bin width is $10 \mathrm{msec}$.

about the same discharge rate at all modulation depths, much like auditory nerve fibers (Fig. $10 \mathrm{~A}$, dashed lines). In contrast, the discharge rate of off neurons increased with modulation depth (Fig. 10B).

Sustained and off neurons also differed in how their synchrony changed with modulation depth. Sustained neurons showed a gradual increase in synchrony with modulation depth that was similar to that of auditory nerve fibers (Fig. 10C, dashed line). The absolute synchrony could be similar or greater than that of auditory nerve fibers. In contrast, the synchrony of off neurons increased rapidly to a high value and then remained constant with modulation depth (Fig. 10D). The synchrony of off neurons was noticeably higher than even the maximum seen in the sample of auditory nerve fibers.

The constancy of the synchrony of off neurons across a wide range of modulation depths implies that the amplification (gain) by these neurons of the modulation in the stimulus changes with increasing depth. This was demonstrated by calculating the modulation gain according to the formula $20 \log 2 R /$ modulation depth (Joris and Yin, 1992), where $R=$ synchrony. At low modulation depths, the gain of off neurons (Fig. $10 \mathrm{~F}$ ) was greater than that of sustained neurons (Fig. 10E), and that of auditory nerve fibers 


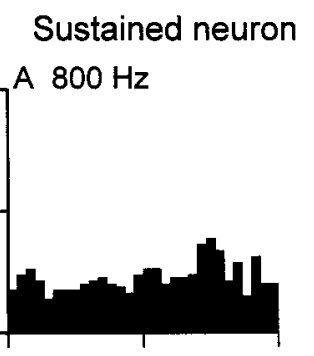

B $400 \mathrm{~Hz}$
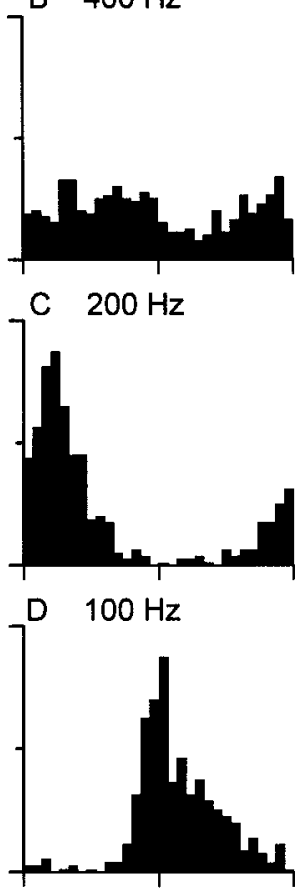

E $50 \mathrm{~Hz}$
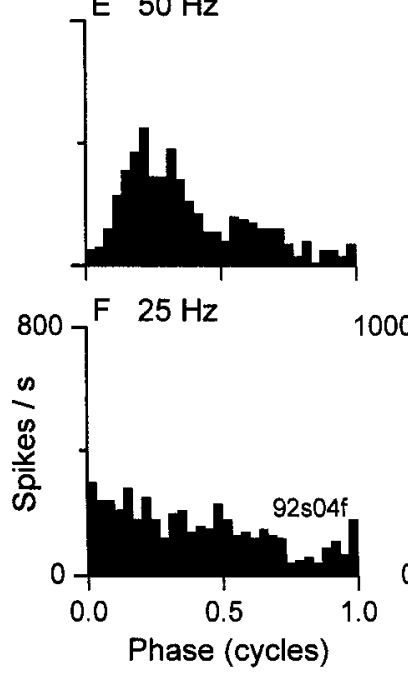

Figure 4. Synchrony of off neurons was generally higher and more constant across modulation frequencies than sustained neurons. $A-F$, Cycle histograms of a sustained neuron at different modulation frequencies (derived from the responses in Fig. $3 A-E$ ). $G-L$, Same for an off neuron (derived from the responses in Fig. $3 F-J$ ). Cycle histograms were created by averaging the responses at the same stimulus modulation phase. All responses showed significant synchrony except $A$. Bin width is 0.033 cycles.
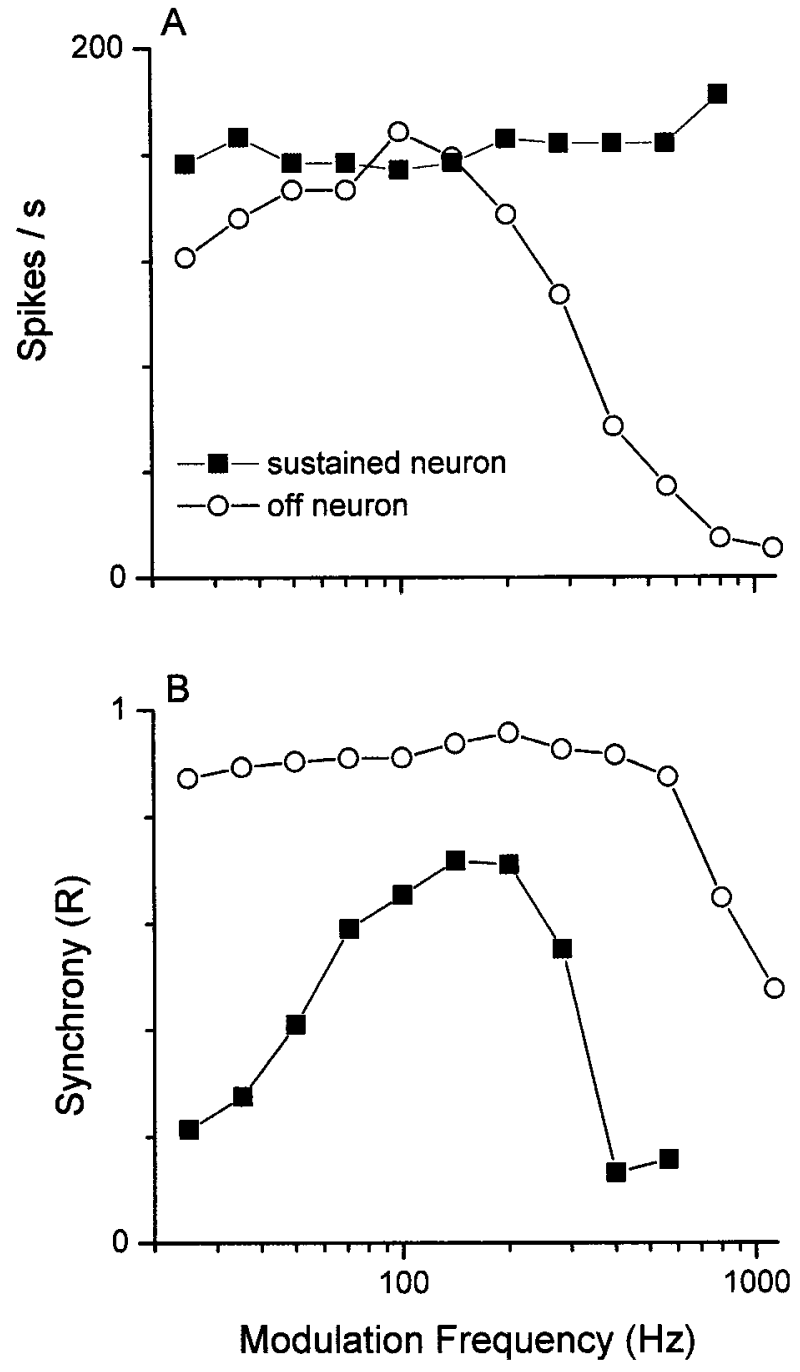

Figure 5. Summary plot of the rate MTFs and synchrony MTFs for the sustained neurons and the off neuron illustrated in Figure 4. A, Plots of discharge rate versus modulation frequency (rate MTFs) for the sustained $(\boldsymbol{\square})$ and off $(\bigcirc)$ responses. $B$, Plots of synchrony versus modulation frequency (synchrony MTFs) for the same two neurons. All discharge rates tested are plotted in $A$, but only responses that showed significant synchrony are displayed in $B$. Spontaneous rates for sustained and off neuron were 94.7 and 56.6 spikes/sec, respectively.

(dashed lines). As the depth was increased, the gain of both sustained and off neurons declined, but the gain of off neurons declined more steeply. At $100 \%$ modulation, the gain of sustained and off neurons was similar and resembled the maximum gain seen in the sample of auditory nerve fibers.

Figure 11 plots the rate and synchrony MTFs at several modulation depths for a sustained (Fig. $11 A, B$ ) and an off neuron (Fig. $11 E, F)$. The rate MTFs of the sustained neuron remained relatively flat and constant at all modulation depths (Fig. 11B). In contrast, the rate MTFs of the off neuron changed with modulation depth $($ Fig. $11 F)$. At lower modulation depths $(<30 \%)$, the functions were flat, and the discharge rate was lower than the spontaneous activity (arrows). This presumably occurred because at lower modulation depths the SAM tones resemble pure tones that suppress neural activity during the stimulation period (e.g., Fig. $1 E-H)$. At higher modulation depths, the discharge rate of off neurons rose above the spontaneous level, and the rate MTFs 

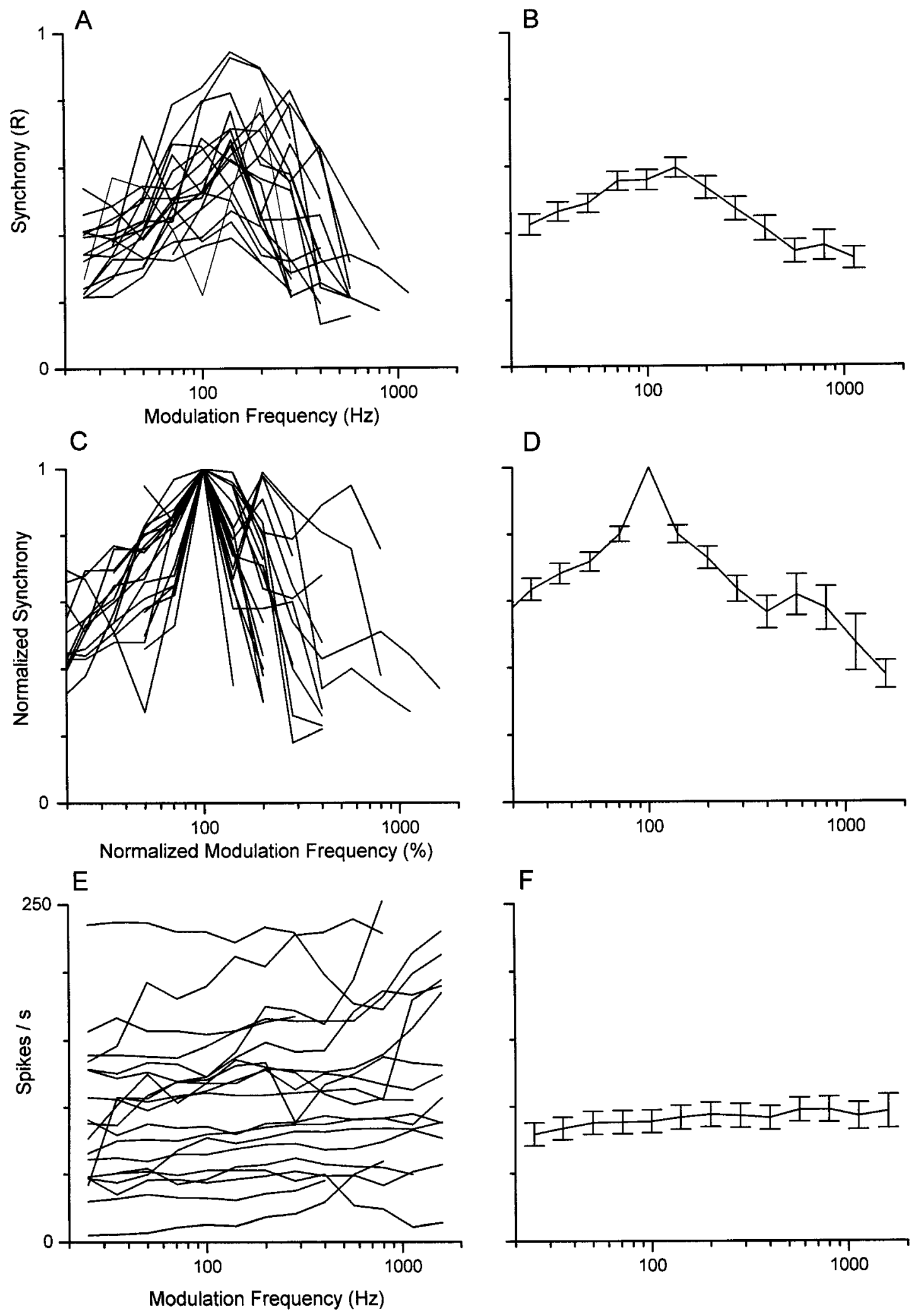

Figure 6. In general, the synchrony MTFs of sustained neurons were bandpass in shape, whereas their rate MTFs were flat. For visual clarity, only the responses of half (20/42) the sustained neurons are shown. $A$, Synchrony MTFs. $B$, Mean synchrony MTF derived by averaging the synchrony MTFs of all $(n=42)$ the sustained neurons. $C$, Normalized synchrony MTFs of responses in $A$. Both synchrony and each neuron's BMF were normalized. $D$, Mean normalized synchrony MTF of all sustained neurons. $E$, Rate MTFs of the neurons in $A$. $F$, Mean rate MTF of all sustained neurons. Error bars represent SE. 

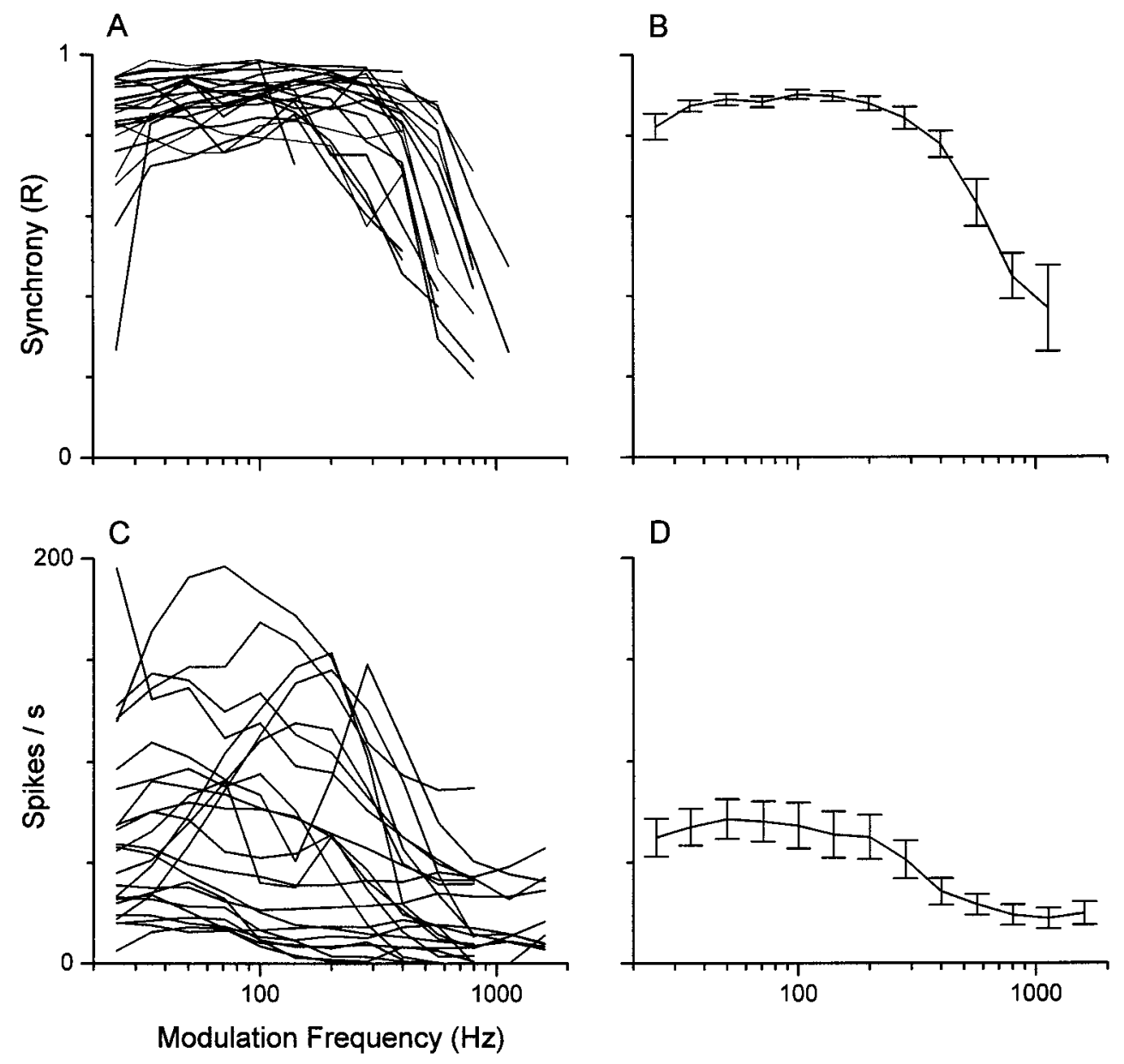

Figure 7. In general, synchrony and rate MTFs of off neurons were low-pass. $A$, Synchrony MTFs of all off neurons $(n=$ 23). $B$, Mean synchrony MTF derived by averaging the synchrony MTFs in $A$. $C$, Rate MTFs of all off neurons. $D$, Mean rate MTF of all off neurons. Error bars represent $\mathrm{SE}$. were low-pass. Note that even at these modulation depths, the response falls below the spontaneous level at high modulation frequencies. However, at lower modulation frequencies, the periodic inhibition to each modulation is completely or nearly completely released, evoking a rebound discharge to each modulation. This results in a discharge rate higher than the spontaneous activity. In contrast, at low modulation depths or high modulation frequencies, the periodic inhibition is never released and consequently there is no rebound response. The inhibition now serves to only attenuate and modulate the spontaneous activity.

The synchrony MTFs of the sustained neuron at different modulation depths resembled a bandpass function (Fig. 11A), and synchrony increased with modulation depth. Although the BMF could shift slightly with modulation depth, in general the function remained bandpass. In comparison, the synchrony MTFs of the off neuron (Fig. 11E) were low-pass and uniformly high, except at the lowest modulation depths (5\%). The lower synchrony at these low modulation depths is likely to be a consequence of the periodic modulation of the spontaneous activity as discussed above.

\section{Effects of stimulus level}

We have already described the dynamic range of neurons in the SOC to short tone bursts (Fig. 2). Here we examine their dynamic range to SAM tones. Figure 12 plots the rate and synchrony of the response as a function of stimulus level for seven sustained and seven off neurons at their BMF. The discharge rate of sustained neurons increased monotonically with a dynamic range typically
$>40 \mathrm{~dB}$ SPL (Fig. 12A). Off neurons usually had a nonmonotonic rate level function (Fig. 12B). The two off neurons in the sample that displayed a monotonic rate-level function (Fig. 12B; neurons 13 and 14) also had a high threshold for synchrony. If we had tested higher stimulus levels on these neurons, the rate-level function might have been nonmonotonic. The monotonic ratelevel functions to tones and nonmonotonic functions to SAM tones displayed by off neurons have also been observed in the MSO of the free-tailed bat (Grothe et al., 1997). A possible explanation for the nonmonotonic behavior may involve the relationship between the latency of the off response and the period of the modulation envelope. As the intensity of a pure tone was increased, the off discharge increased (Fig. 2), as well as its latency (data not shown). For low and intermediate intensity SAM tones, the off response to one cycle of modulation may not interact with the suppression produced by the next cycle. However, at higher intensities where the latency is longer, the off response to one cycle may be attenuated by the suppression evoked by the next cycle. In this way, the SAM tone behavior becomes nonmonotonic.

In contrast to the rate-level function, the synchrony-level function of sustained neurons took many forms. Synchrony could remain relatively constant (Fig. $12 C$, neuron 6 ), increase (neuron 2), decrease (neurons 1,3,7), or be nonmonotonic (neurons 4, 5) with stimulus level. In comparison, the synchrony of most off neurons increased rapidly with stimulus level (Fig. 12D), saturating at a value of $\sim 0.9$ by $\sim 20 \mathrm{~dB}$ SPL. 
A
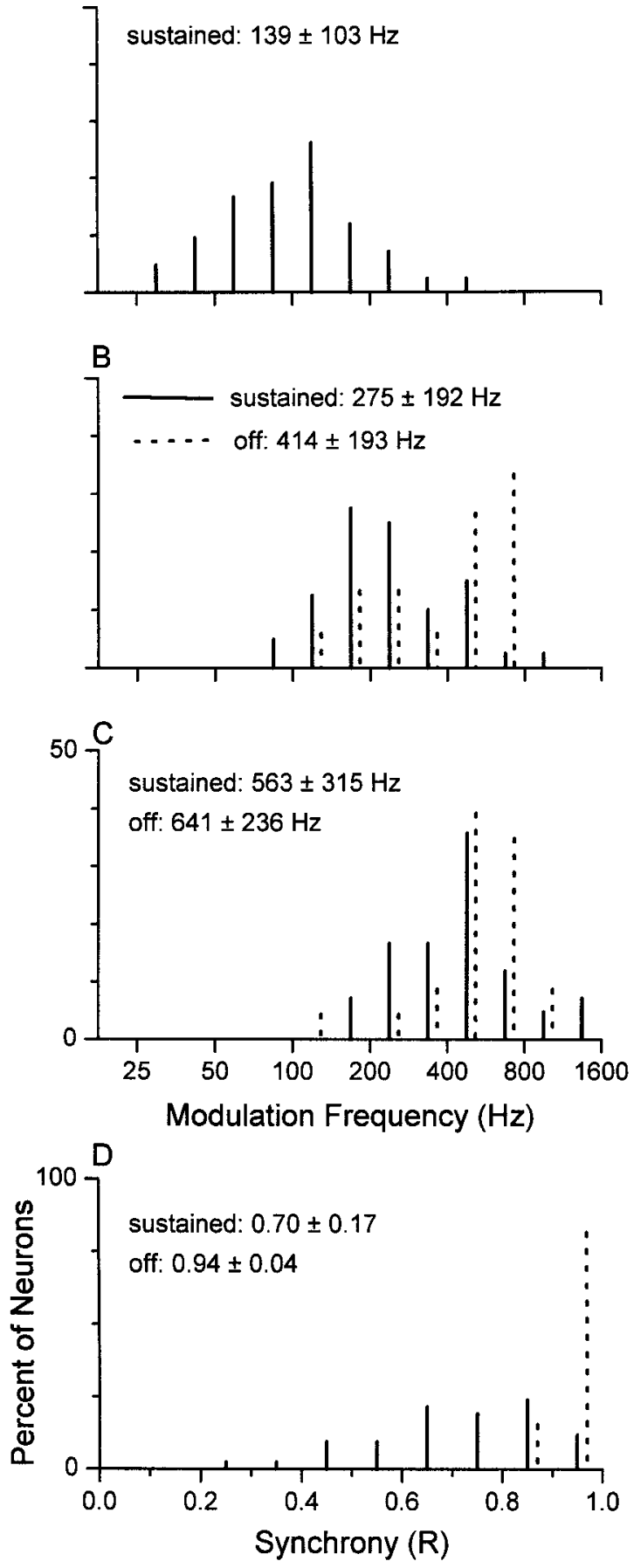

Figure 8. Off neurons encoded a slightly higher range of modulation frequencies than sustained neurons. $A$, Distribution of BMF for sustained $(n=42)$ neurons. $B$, Distribution of upper cutoff frequencies for sustained $(n=40)$ and off $(n=15)$ neurons. The upper cutoff was the modulation frequency above the neuron's BMF at which the synchrony was $20 \%$ lower than the maximum. $C$, Distribution of highest modulation frequency where significant synchrony was still present for sustained $(n=42)$ and off $(n=23)$ neurons. $D$, Off neurons synchronized more strongly than sustained neurons. For each neuron, the highest synchrony across modulation frequency, modulation depth, and sound intensity was selected. Shown is the distribution of this synchrony for sustained $(n=42)$ and off $(n=23)$ neurons. For $A-C$, bin width is 0.5 octaves; for $D$ it is 0.1 cycles. Solid lines (sustained neurons) were centered on each bin, and dashed lines (off neurons) have been shifted slightly rightward for visual clarity. For each distribution, the mean and SD for sustained and off neurons is indicated.
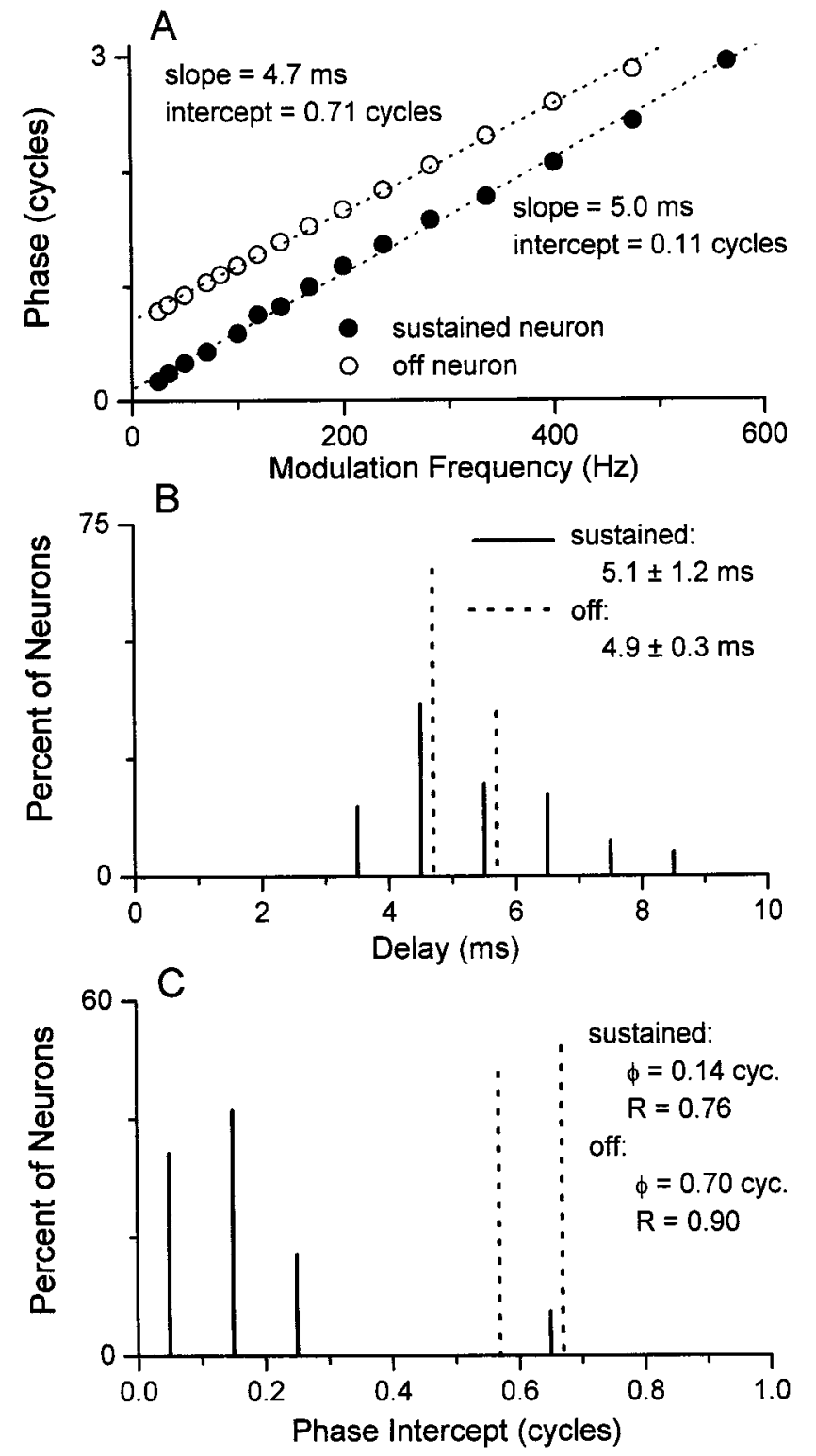

Figure 9. Sustained and off neurons had similar delays but different phase intercepts. $A$, Phase versus frequency plots for a sustained $(\bullet)$ and an off $(\bigcirc)$ neuron. The plot for each neuron is fit with a straight line using a least-squares criterion. The slope of this line is an estimate of the delay between the sound source and the neural response. Slope (delay) and phase intercept for the two neurons are as indicated. $B$, Distribution of delays for sustained and off neurons. Bin width is $1 \mathrm{msec}$. Mean delay and $\mathrm{SD}$ are as indicated. $C$, Distribution of phase intercepts for sustained and off neurons. Bin width is 0.1 cycles. Vector mean phase $(\phi)$ and synchrony $(R)$ are as indicated. In both $B$ and $C$, solid lines (sustained neurons) reflect the center of each bin and dashed lines (off neurons) have been shifted slightly rightward for visual clarity. Distributions are based on 41 sustained and 23 off neurons.

The shape of the synchrony MTFs of a sustained neuron could change when the stimulus intensity level was changed (Figs. 11C), whereas that of an off neuron remained relatively constant (Fig. $11 G)$. At the lowest intensity, the synchrony MTFs of the sustained neuron were low-pass (Fig. 11C). However, they became more and more bandpass with increasing stimulus levels. In comparison, the synchrony MTFs of the off neuron were low-pass at all stimulus levels (Fig. 11G). The lower synchrony at low 

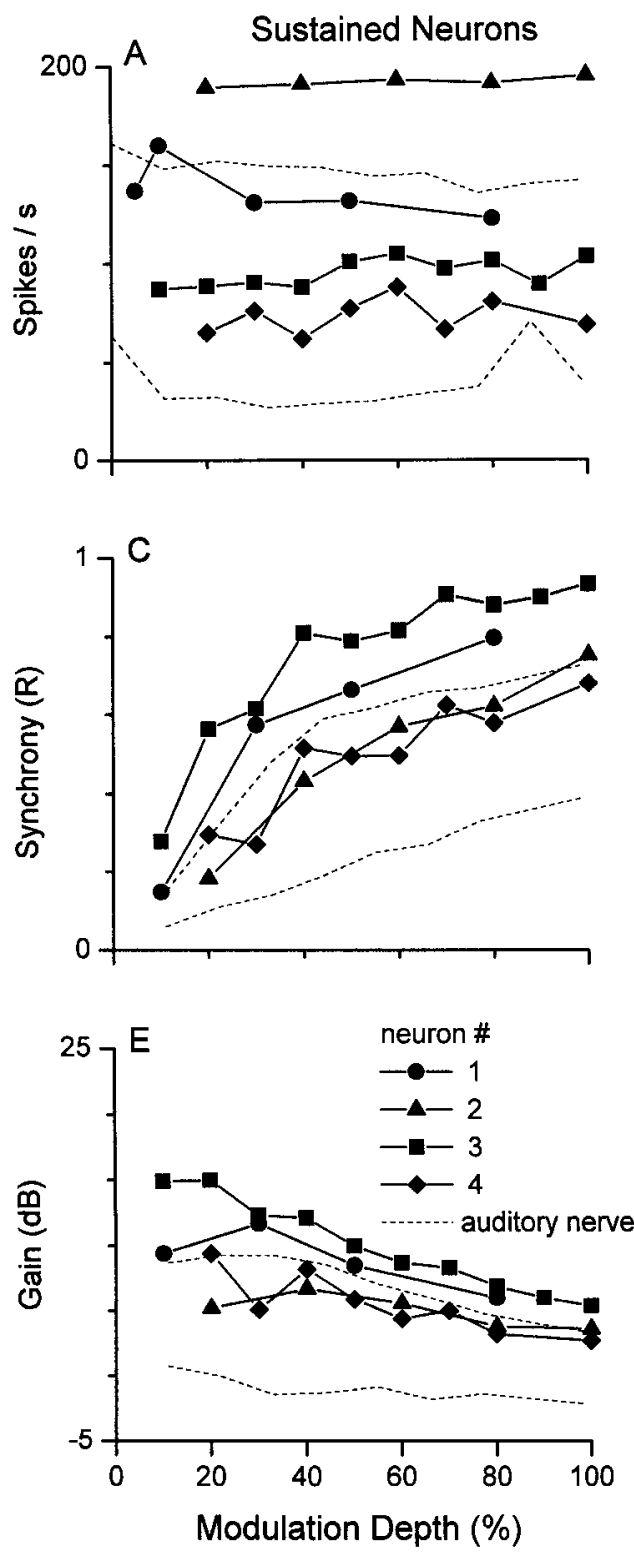
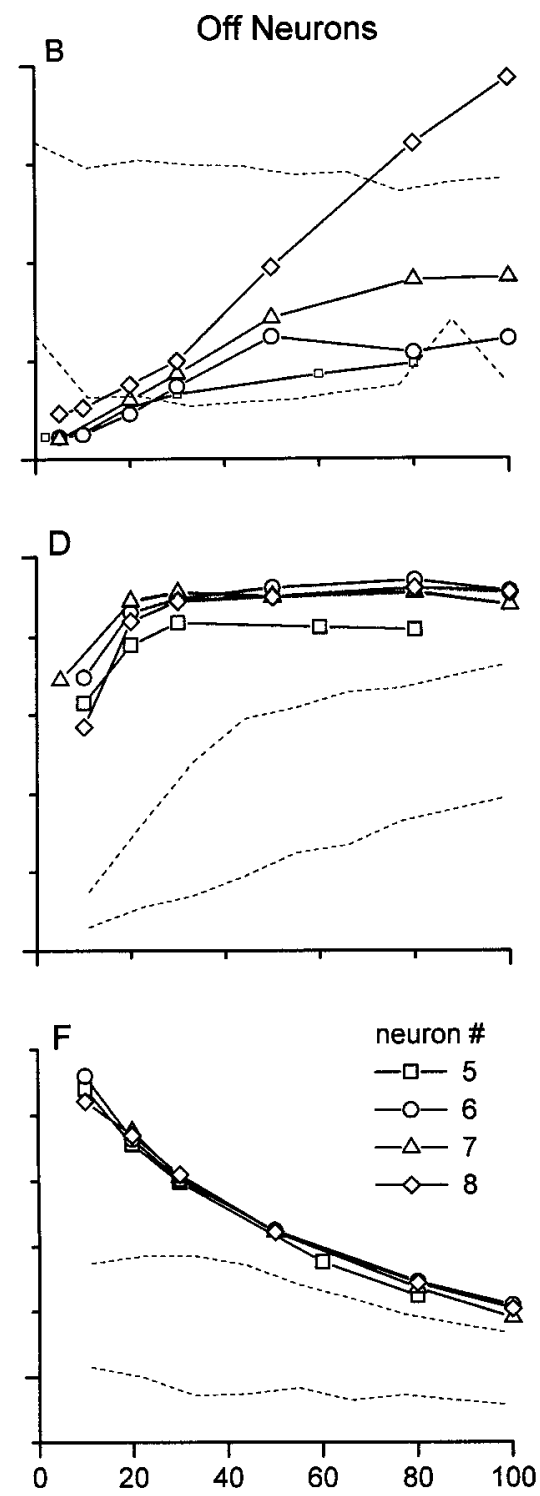

Figure 10. Effects of changing modulation depth on the responses of sustained and off neurons to SAM tones. $A, B$, Discharge rate as a function of modulation depth for four sustained neurons $(A)$ and four off neurons $(B)$. For comparison, the responses of auditory nerve fibers (dashed line) (from Joris and Yin, 1992, their Fig. 2) are provided in all panels. $C, D$, Synchrony as a function of modulation depth for the same four sustained and off neurons as in $A$ and $B$, respectively. $E, F$, Gain as a function of modulation depth for the same four sustained and off neurons. The modulation frequency was set at each neuron's BMF. stimulus levels reflect a modulation of spontaneous activity, where an off response was not present.

The rate MTFs for the sustained neuron remained relatively flat with increasing stimulus levels (Fig. 11D). Where the rate was above spontaneous, the rate MTFs of off neurons were low-pass or mildly bandpass (Fig. $11 H$ ).

\section{DISCUSSION}

Our results demonstrate that sustained and off neurons differ in their ability to follow the pattern of sounds. Somewhat surprisingly, off neurons were better at this than sustained neurons. This indicates that an inhibitory rebound mechanism by itself is sufficient to encode, with high fidelity, ongoing envelopes in complex sounds.

\section{Location of sustained and off neurons}

The locations of sustained and off neurons in the SOC of the rabbit, as shown here, are consistent with those reported in the cat by Guinan et al. (1972a,b), who found that monaural, contralaterally excited neurons (their class 4 ) were located ventro- medial to a line approximately through the center of the MSO. Monaural, ipsilaterally excited neurons (their class 3) were most often located dorsolateral to the MSO. Tsuchitani (1977) also reported that ipsilaterally excited neurons were all located lateral to the MSO. These locations are consistent with the locations of our sustained neurons. Guinan et al. (1972a,b) found that contralaterally evoked off responses (their class 2) were located in the medial nucleus of the trapezoid body (MNTB), ventral nucleus of the trapezoid body, and dorsal medial periolivary nucleus (DMPO). These locations are consistent with Tsuchitani (1977), who found that there were no off responses in cells located lateral to the MSO, and with the present results. Off neurons are also present in the SOC of the mustached bat but are located in the MSO (Grothe, 1994). The MSO of this bat appears specialized for monaural processing related to echolocation (Covey and Casseday, 1991). Perhaps off neurons reflect a general class of neurons that are represented in different structures depending on the specializations of the species. 


\section{Sustained Neuron}
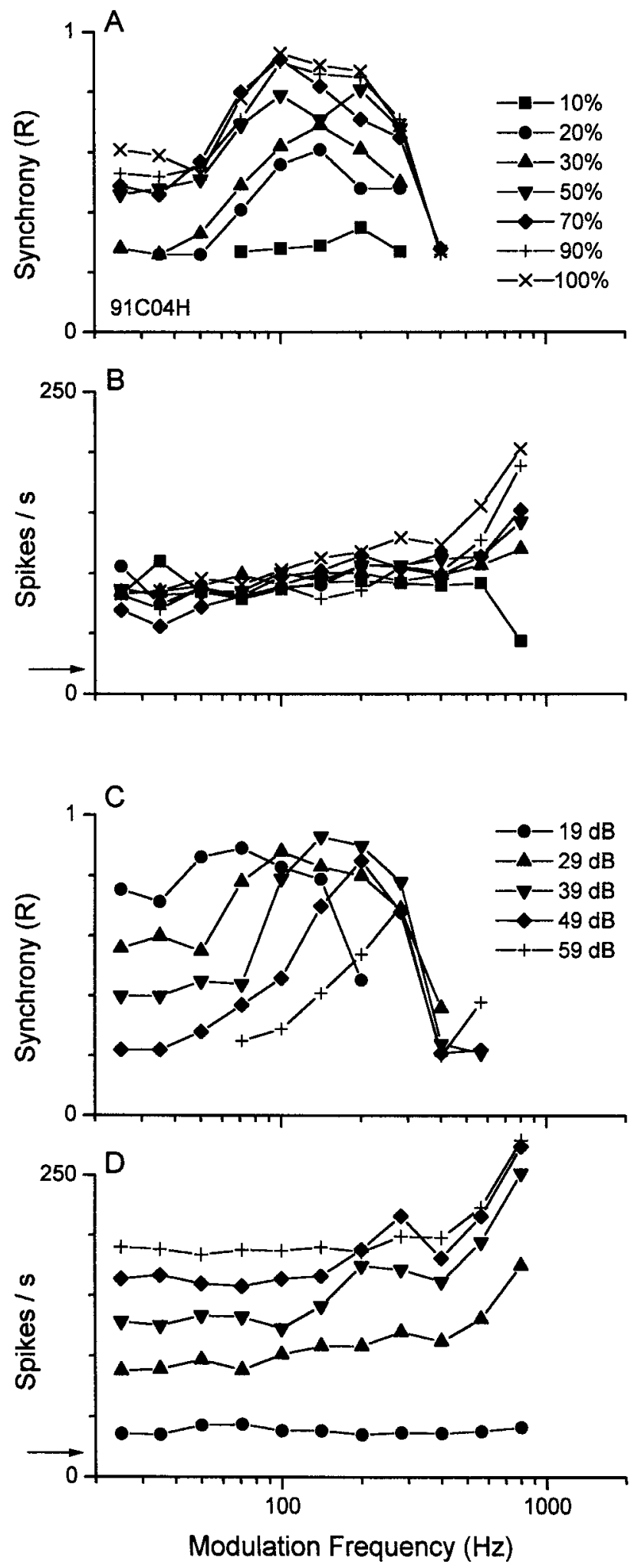

Off Neuron
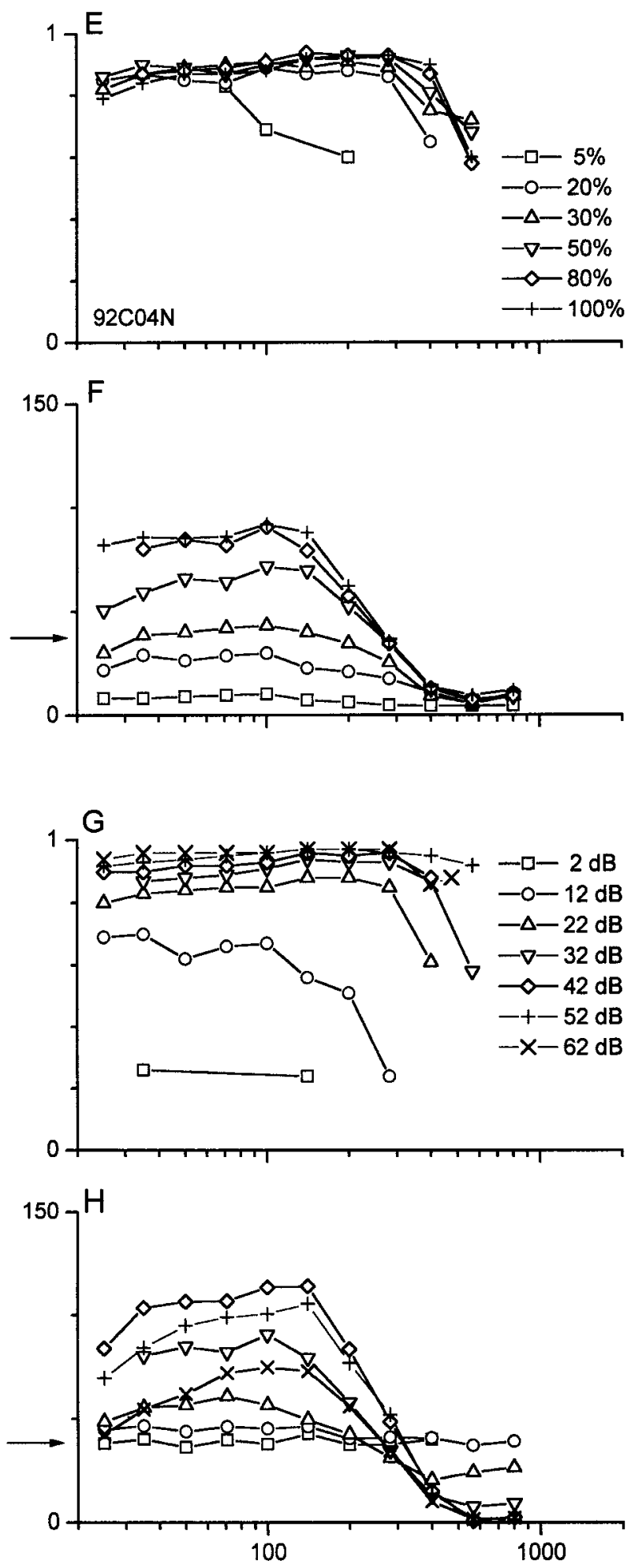

Figure 11. Synchrony and rate MTFs for a sustained and off neuron as a function of modulation depth $(A, B$ and $E, F)$ and stimulus level $(C, D$ and $G, H)$. The shape of the synchrony and rate MTFs of sustained $(A, B)$ and off $(E, F)$ neurons remained relatively constant across modulation depths, but the synchrony MTF of sustained neurons could change with level. Carrier frequency was at the neuron's best frequency. Intensity of the SAM tone for the sustained neuron $(A, B)$ was $29 \mathrm{~dB}$ SPL, and for the off neuron $(E, F)$ it was $28 \mathrm{~dB}$ SPL. Depth of modulation of the SAM tone for $C, D$, $G$, and $H$ was $80 \%$. Horizontal arrows indicate spontaneous activity (9.7 and 39.1 spikes/sec for spontaneous and off neuron, respectively). 

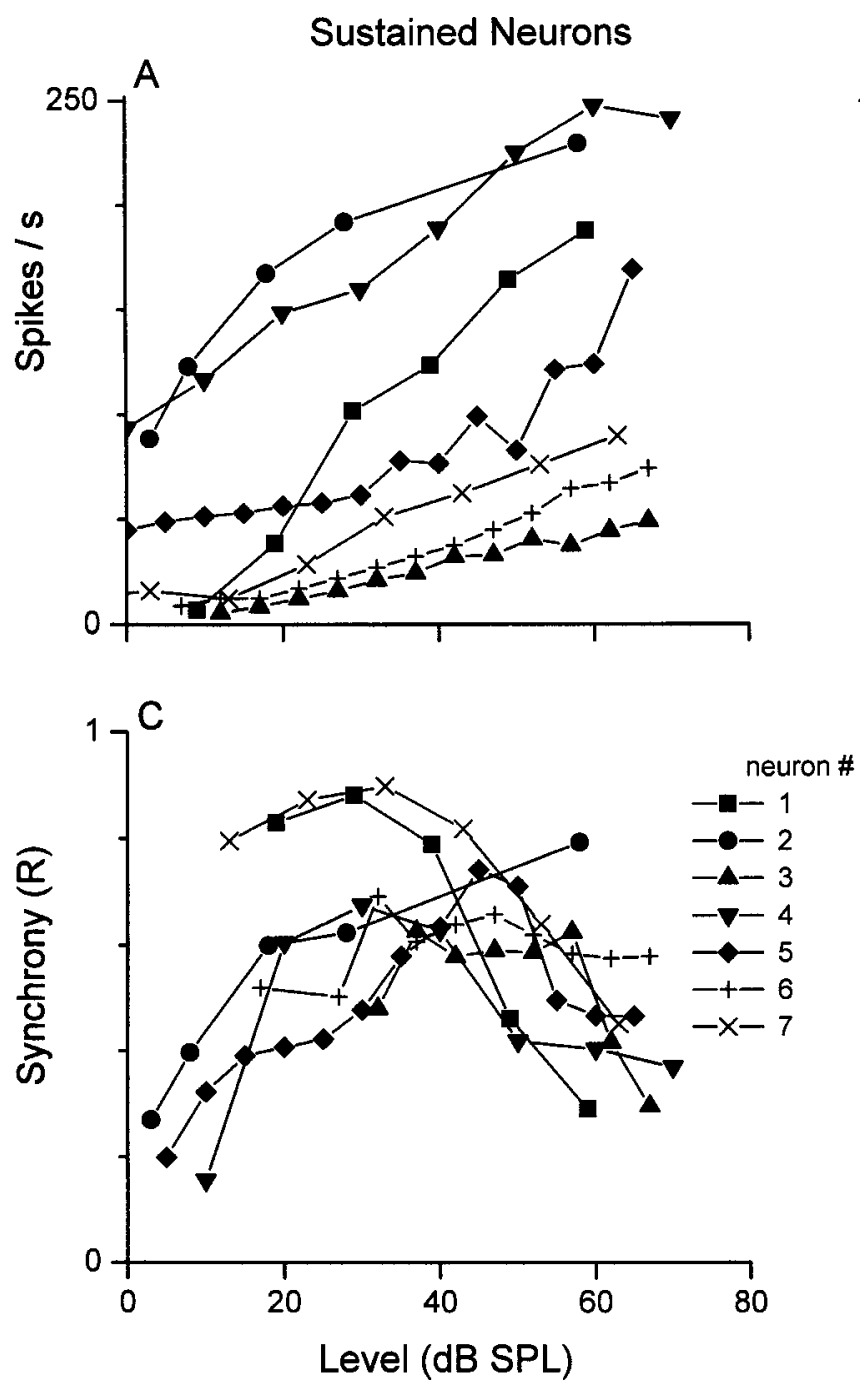
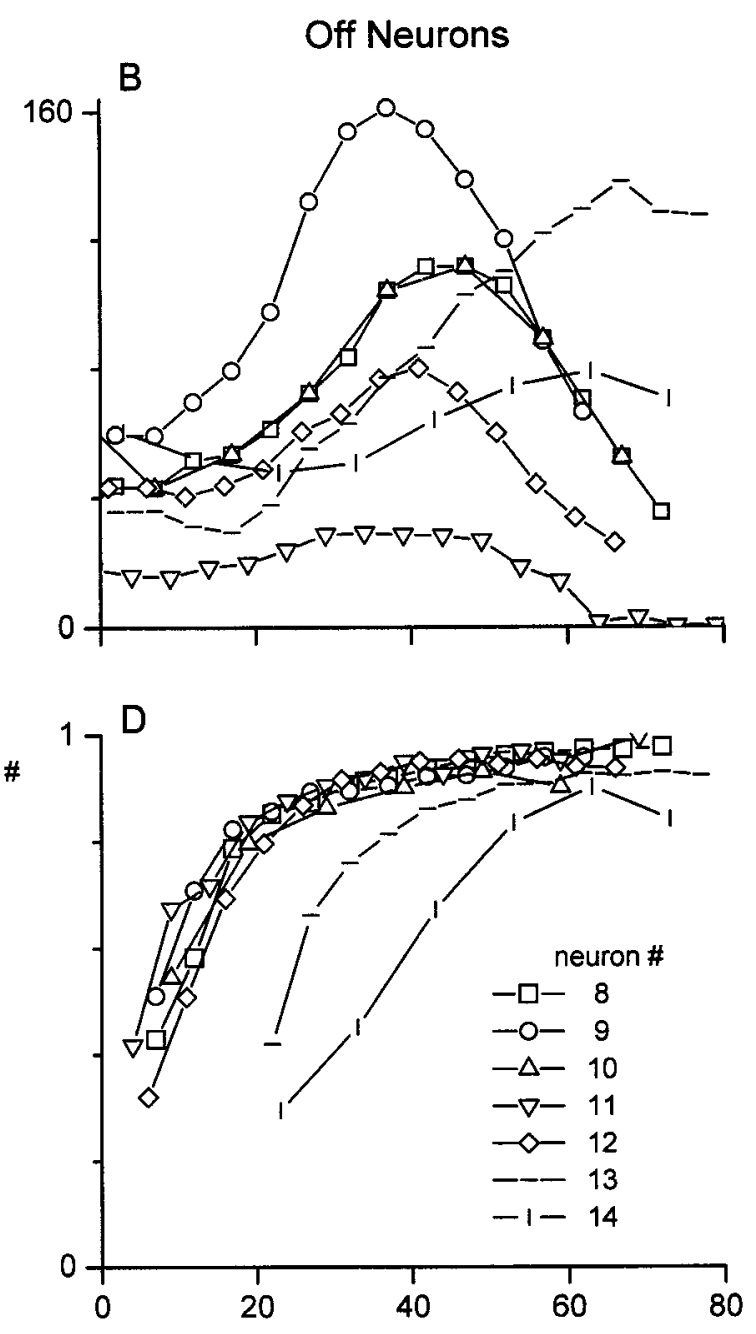

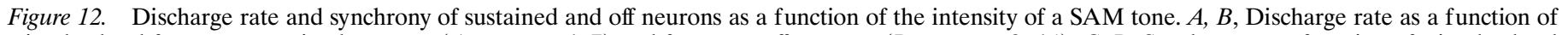

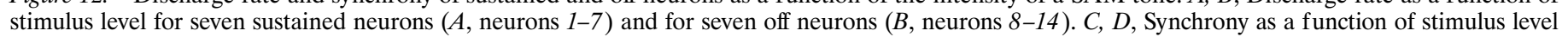

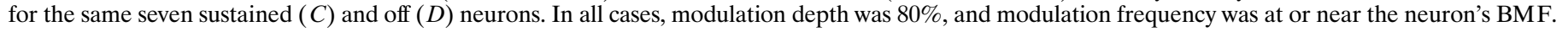

\section{Response to tones}

The short delays of our sustained and off neurons suggest that their inputs are from $\mathrm{CN}$ neurons or from other SOC neurons. Their large dynamic ranges suggest that they receive inputs from many $\mathrm{CN}$ neurons, or from a select population of cells in the $\mathrm{CN}$ [e.g., onset choppers (Rhode and Greenberg, 1994b); neurons in the small cell cap (Ghoshal and Kim, 1996)]. The dynamic range of off neurons in the SOC has not been studied previously. Tsuchitani (1977) reported that the dynamic range of monaural sustained neurons in the lateral cell group was $\sim 12-80 \mathrm{~dB}$, a range similar to that found here.

Our off neurons probably correspond to three different categories of neurons described by Guinan et al. (1972a). Their off category (class 2 ) has disparities with ours. They included in their off category only neurons that had a short-latency, tightly locked response. Other neurons that had a long-lasting, long-latency off response were assigned to different categories (classes 9 and 10). Our off neurons had short latencies (as measured by the phase delay), like those in their class 2, but also had a long-lasting response, like those in their classes 9 and 10. They used a barbiturate-anesthetized preparation, whereas our preparation was anesthetic-free. Because barbiturates are known to potentiate GABA-mediated inhibition (Barker and Ransom, 1978) and GABAergic terminals and neurons are present in the SOC (Wenthold, 1991), it is possible that their classes 2, 9, and 10 represent a single class of off neurons. Consistent with this idea, the locations of their classes 9 and 10 neurons overlapped with that of their class 2 neurons.

\section{Responses to SAM tones}

The responses of sustained neurons in the SOC to SAM tones could simply be a reflection of the input from the ventral $\mathrm{CN}$. Most of the MTFs of our regular, sustained neurons (Fig. 6A) resembled those of regular, sustained neurons in the $\mathrm{CN}$. There, sustained neurons have synchrony MTFs with a bandpass shape that becomes more low-pass at lower stimulus intensities (Frisina et al., 1990; Rhode and Greenberg, 1992). We have also shown that some sustained neurons may have a higher modulation gain than the auditory nerve. Some neurons of CN appear to have a similar increase in gain (Frisina et al., 1990). Thus, there appears to be no substantial increase in gain between the $\mathrm{CN}$ and the sustained neurons of the SOC. 
In contrast to sustained neurons, the responses of off neurons to SAM tones did not appear to be a mere reflection of CN input. The gain of off neurons was much higher than that of most neurons in the $\mathrm{CN}$, particularly at shallow modulation depths. Similar results have been reported for off neurons in the MSO of the mustached bat (Grothe, 1994). As in the rabbit, off neurons in the bat MSO synchronized strongly to envelopes and had synchrony and rate MTFs that were low-pass.

The MTFs of binaural neurons in the LSO have also been examined (Batra et al., 1997b; Joris and Yin, 1998). Like the off neurons, the sustained neurons of the LSO displayed high synchrony and synchrony and rate MTFs that were low-pass. Moreover, in general their synchrony was higher than the sustained neurons of this study. The high synchrony of LSO neurons may be a consequence of an ipsilateral inhibitory input (Brownell et al., 1979; Kuwabara and Zook, 1992) that would serve to increase synchrony (Grothe, 1994).

\section{Neural mechanisms of off neurons}

The off response is most likely a rebound from inhibition, rather than an excitatory response that has been partially occluded by inhibition during the stimulus. Off neurons in the MSO of the bat are monaural and receive excitatory and inhibitory inputs from the contralateral side (Grothe, 1994). Inactivating the inhibitory input pharmacologically revealed that the off response was separate from the excitatory response during the stimulus. Our finding that the off neurons discharge near the trough of the modulation cycle (Figs. 9A,D) is also consistent with an inhibitory rebound mechanism.

The source of the inhibitory input to off neurons is probably not the ventral $\mathrm{CN}$ because it has very few glycinergic or GABAergic neurons (Peyret et al., 1986, 1987). The short delay of the off response suggests that the source is within the SOC itself. One possible source for this inhibitory input is the principal neurons of the MNTB. These neurons give off collaterals within the MNTB itself, as well as in the DMPO (Morest, 1968; Banks and Smith, 1992; Kuwabara and Zook, 1992). Stellate cells in the MNTB are thought to be one of the sources of off responses as are the neurons in the DMPO (Guinan et al., 1972b). The observation that the principal neurons of the MNTB, like our off neurons, have low-pass synchrony MTFs is also consistent with the notion that these neurons are the source of inhibition for off neurons (Joris and Yin, 1998).

Inhibitory rebound by itself appears sufficient to explain the strong off responses we encountered, but in other neurons in the CNS it usually evokes only an action potential or two. Recently, Wu and Kelly (1995) reported that neurons in the dorsal nucleus of the lateral lemniscus produced a long-lasting depolarization in response to the release of hyperpolarization. This depolarization could elicit a burst of action potentials. Perhaps this membrane characteristic is also present in the off neurons in the SOC.

Why is synchrony of off neurons so high? Grothe (1994) suggested that the rebound response to the release of inhibition attributable to the falling slope of the modulation envelope is sharpened by the increasing inhibition created by the rising slope of the next envelope. The upper cutoff frequency of off neurons is probably related to the time course of the release from inhibition. At low modulation frequencies, the magnitude of the rebound response is only marginally reduced by the next modulation envelope because the modulation period is long. However, at high modulation frequencies, the rebound response is markedly reduced by the subsequent envelope because the period is short.
Synchrony would remain high or even increase. Ultimately, there would be no rebound response because the time course of the release from inhibition would overlap with the rising inhibition to the next modulation envelope.

\section{Functional considerations}

In general, the rate MTFs of sustained and off neurons were flat and low-pass, respectively. Thus, there is little, if any, information about modulation frequency conveyed in the discharge rate. However, the discharge rate of sustained neurons displayed a monotonic increase with intensity to SAM tones, suggesting a role in intensity coding of complex signals and pure tones.

The synchrony of sustained neurons often peaked at a particular modulation frequency, suggesting that such neurons could optimally convey information about that modulation frequency using a temporal code. However, the BMF often changed as a function of level. Furthermore, at low levels the synchrony MTFs were low-pass, and a BMF was not present. In contrast, the synchrony of off neurons was high and constant across a wide range of modulation frequencies and levels. Because off neurons can discharge to each cycle of the modulation, independent of stimulus level, they are ideal candidates to transmit precise temporal information about envelopes to higher centers. Whether their influence on neurons in higher centers is excitatory or inhibitory is unknown, but in either case they could serve to shape the way that information about envelopes is encoded by the system.

\section{REFERENCES}

Anderson D, Rose J, Hind JE, Brugge JF (1971) Temporal position of discharges in single auditory nerve fibers within the cycle of a sine-wave stimulus: frequency and intensity effects. J Acoust Soc Am 49:1131-1139.

Banks MI, Smith PH (1992) Intracellular recordings from neurobiotinlabeled cells in brain slices of the rat medial nucleus of the trapezoid body. J Neurosci 12:2819-2837.

Barker JL, Ransom BR (1978) Pentobarbitone pharmacology of mammalian central neurones grown in tissue culture. J Physiol (Lond) 280:355-372.

Batra R, Kuwada S, Stanford TR (1989) Temporal coding of envelopes and their interaural delays in the inferior colliculus of the unanesthetized rabbit. J Neurophysiol 61:257-268.

Batra R, Kuwada S, Fitzpatrick DC (1997a) Sensitivity to interaural temporal disparities of low- and high-frequency neurons in the superior olivary complex. I. Heterogeneity of responses. J Neurophysiol 78:1222-1236.

Batra R, Kuwada S, Fitzpatrick DC (1997b) Sensitivity to interaural temporal disparities of low- and high-frequency neurons in the superior olivary complex. II. Coincidence detection. J Neurophysiol 78:1237-1247.

Brownell WE, Manis PB, Ritz LA (1979) Ipsilateral inhibitory responses in the cat lateral superior olive. Brain Res 177:189-193.

Covey E, Casseday JH (1991) The monaural nuclei of the lateral lemniscus in an echolocating bat: parallel pathways for analyzing temporal features of sound. J Neurosci 11:3456-3470.

Creutzfeldt O, Hellweg F-C, Schreiner C (1980) Thalamocortical transformations of responses to complex auditory stimuli. Exp Brain Res 39:87-104.

Eggermont JJ (1993) Differential effects of age on click-rate and amplitude modulation-frequency coding in primary auditory cortex of the cat. Hear Res 65:175-192.

Evans EF, Nelson PG (1973) The responses of single neurons in the cochlear nucleus of the cat as a function of their location and anesthetic state. Exp Brain Res 117:402-427.

Frisina RD, Smith RL, Chamberlin SC (1990) Encoding of amplitude modulation in the gerbil cochlear nucleus: I. A hierarchy of enhancement. Hear Res 44:99-122.

Glendenning KK, Masterton RB (1995) Comparative morphometry of mammalian central auditory system: variation in nuclei and form of the ascending system. Brain Behav Evol 51:59-89. 
Goldberg JM, Brown PB (1969) Response properties of binaural neurons of dog superior olivary complex to dichotic tonal stimuli: some physiological mechanisms of sound localization. J Neurophysiol 32:613-636.

Ghoshal S, Kim DO (1996) Marginal shell of the anteroventral cochlear nucleus: intensity coding in single units of the unanesthetized, decerebrate cat. Neurosci Lett 205:71-74.

Grothe B (1994) Interaction of excitation and inhibition in processing of pure tone and amplitude-modulated stimuli in the medial superior olive of the mustached bat. J Neurophysiol 71:706-721.

Grothe B, Park TJ, Schuller G (1997) Medial superior olive in the free-tailed bat: responses to pure tones and amplitude-modulated tones. J Neurophysiol 77:1553-1565.

Guinan JJ, Guinan SS, Norris BE (1972a) Single auditory units in the superior olivary complex I: responses to sounds and classifications based on physiological properties. Int J Neurosci 4:101-120.

Guinan JJ, Norris BE, Guinan SS (1972b) Single auditory units in the superior olivary complex II: locations of unit categories and tonotopic organization. Int J Neurosci 4:147-166.

Heil P, Schultz H, Langner G (1995) Ontogenetic development of periodicity coding in the inferior colliculus of the mongolian gerbil. Auditory Neurosci 1:363-383.

Helfert RH, Aschoff A (1997) Superior olivary complex and nuclei of the lateral lemniscus. In: The central auditory system (Erhet G, Romand R, eds), pp 193-258. New York: Oxford UP.

Joris PX, Yin TCT (1992) Responses to amplitude-modulated tones in the auditory nerve of the cat. J Acoust Soc Am 91:215-232.

Joris PX, Yin TCT (1998) Envelope coding in the lateral superior olive. III. Comparison with afferent pathways. J Neurophysiol 79:253-269.

Kim DO, Sirianni JG, Change SO (1990) Responses of DCN-PVCN neurons and auditory nerve fibers in unanesthetized decerebrate cats to AM and pure tones: analysis with autocorrelation/power-spectrum. Hear Res 45:95-113.

Kuwabara N, Zook JM (1992) Projections of the medial superior olive from the medial and lateral nuclei of the trapezoid body in rodents and bats. J Comp Neurol 324:522-538.

Kuwada S, Yin TCT, Wickesberg RE (1979) Response of cat inferior colliculus neurons to binaural beat stimuli: possible mechanisms for sound localization. Science 206:586-588.

Kuwada S, Stanford TR, Batra R (1987) Interaural phase-sensitive units in the inferior colliculus of the unanesthetized rabbit: effects of changing phase. J Neurophysiol 57:1338-1360.

Kuwada S, Batra R, Stanford TR (1989) Monaural and binaural response properties of neurons in the inferior colliculus of the rabbit: effects of sodium pentobarbital. J Neurophysiol 61:269-282.

Langner G, Schreiner CE (1988) Periodicity coding in the inferior colliculus of the cat. I. Neuronal mechanisms. J Neurophysiol 60:1799-1822.

Liberman MS, Brown ME (1986) Physiology and anatomy of single olivocochlear neurons in the cat. Hear Res 24:17-36.

Mardia KV (1972) Statistics of directional data. New York: Academic.

Moller AR (1974) Coding of sounds with rapidly varying spectrum in the cochlear nucleus. J Acoust Soc Am 55:631-640.

Moller AR (1976) Dynamic properties of the responses of single neurones in the cochlear nucleus of the rat. J Neurophysiol 259:63-82.

Morest DK (1968) The growth of synaptic endings in the mammalian brain: a study of the calyces of the trapezoid body. Z Anat Entwicklungsgesch 127:201-220.

Palmer AR (1982) Encoding of rapid amplitude fluctuations by cochlearnerve fibers in the guinea pig. Arch Otorhinolaryngol 236:197-202.
Peyret D, Geffard M, Aran JM (1986) GABA immunoreactivity in the primary nuclei of the auditory central nervous system. Hearing Res 23:115-121.

Peyret D, Campistron G, Gelfard M, Aran J-M (1987) Glycine immunoreactivity in the brainstem auditory and vestibular nuclei of the guinea pig. Acta Otolaryngol (Stockh) 104:71-76.

Ramón y Cajal S (1909) Histologie du Système Nerveux de l'Homme et des Vertébrés, Vol 1. Madrid, Instituto Ramon y Cajal. Paris: Maloine, 1972.

Rees A, Moller AR (1987) Stimulus properties influencing the responses of inferior colliculus neurons to amplitude-modulated sounds. Hear Res 27:129-143.

Rees A, Palmer AR (1989) Neuronal responses to amplitude-modulated and pure-tone stimuli in the guinea pig inferior colliculus, and their modification by broadband noise. J Acoust Soc Am 85:1978-1994.

Rhode WS (1976) A digital system for auditory neurophysiological research. In: Current computer technology in neurobiology (Brown P, ed) pp 543-567. Washington, DC: Hemisphere.

Rhode WS (1994) Temporal coding of $200 \%$ amplitude modulated signals in the ventral cochlear nucleus of cat. Hearing Res 77:43-68.

Rhode WS, Greenberg S (1992) Physiology of the cochlear nuclei. In: The mammalian auditory pathway: neurophysiology, Ed 1, Vol 2 (Popper AN, Fay RR, eds), pp 94-152. New York: Springer.

Rhode WS, Greenberg S (1994a) Encoding of amplitude modulation in the cochlear nucleus of the cat. J Neurophysiol 71:1797-1825.

Rhode WS, Greenberg S (1994b) Lateral suppression and inhibition in the cochlear nucleus of the cat. J Neurophysiol 71:493-514.

Ritz LA, Brownell WE (1982) Single unit analysis of the posteroventral cochlear nucleus of the decerebrate cat. Neuroscience 7:1995-2010.

Rouiller E, de Ribaupierre Y, Toros-Morel A, de Ribaupierre F (1981) Neural coding of repetitive clicks in the medial geniculate body of the cat. Hear Res 11:235-247.

Schreiner CE, Urbas JV (1986) Representation of amplitude modulation in the auditory cortex of the cat. I. The anterior auditory field (AAF). Hear Res 21:227-241.

Schreiner CE, Urbas JV (1988) Representation of amplitude modulation in the auditory cortex of the cat. II. Comparison between cortical fields. Hear Res 32:49-64.

Schwartz IR (1992) The superior olivary complex and lateral lemniscal nuclei. In: The mammalian auditory pathway: neuroanatomy, Vol 1 (Webster DB, Popper AN, Fay RR, eds), pp 117-167. New York: Springer.

Tsuchitani C (1977) Functional organization of the lateral cell groups of the cat superior olivary complex. J Neurophysiol 40:296-318.

Warr WB (1992) Organization of the olivocochlear efferent system in mammals. In: The mammalian auditory pathway: neuroanatomy (Webster DB, Popper AN, Fay RR, eds), pp 410-448. New York: Springer.

Wenthold RJ (1991) Neurotransmitters of brainstem auditory nuclei. In: Neurobiology of hearing: the central auditory system (Altschuler RA, Bobbin RP, Clopton BM, Hoffman DW, eds), pp 121-140. New York: Raven.

Wu S, Kelly JB (1995) In vitro brain slice studies of the rat's dorsal nucleus of the lateral lemniscus. I. Membrane and synaptic response properties. J Neurophysiol 73:780-793.

Young ED, Brownell WE (1976) Response to tones and noise of single cells in dorsal cochlear nucleus of unanesthetized cats. J Neurophysiol 39:282-300. 